\title{
Análisis de la evolución temática de la investigación sobre Información y Documentación en español en la base de datos LISA mediante modelado temático (1978-2019)
}

\author{
Assessment of the thematic evolution of Library and \\ Information Science (LIS) research in Spanish in the \\ LISA database using latent Dirichlet allocation (LDA)
}

\section{Francisco-Javier García-Marco; Carlos G. Figuerola; María Pinto}

Cómo citar este artículo:

García-Marco, Francisco-Javier; Figuerola, Carlos G.; Pinto, María (2020). “Análisis de la evolución temática de la investigación sobre Información y Documentación en español en la base de datos LISA mediante modelado temático (1978-2019)". Profesional de la información, v. 29, n. 4, e290427.

https://doi.org/10.3145/epi.2020.jul.27

Artículo recibido el 24-12-2019

Aceptación definitiva: 04-06-2020

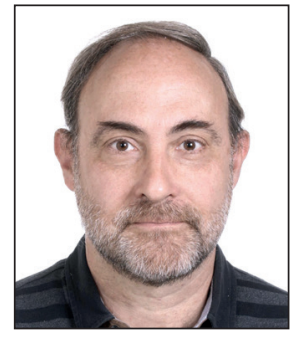

Francisco-Javier García-Marco https://orcid.org/0000-0002-6241-4060

Universidad de Zaragoza

Instituto de Patrimonio y Humanidades

Pedro Cerbuna, 12

50009 Zaragoza, España

jgarcia@unizar.es

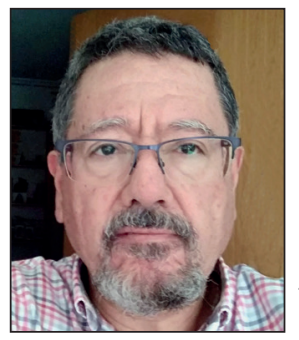

Carlos G. Figuerola

https://orcid.org/0000-0001-6799-2874

Universidad de Salamanca

Departamento de Informática

Plaza de los Caídos, s/n.

37008 Salamanca, España

figue@usal.es

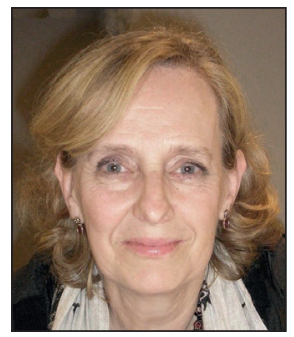

\author{
María Pinto \\ https://orcid.org/0000-0002-5928-6483 \\ Universidad de Granada \\ Facultad de Comunicación y Documentación \\ Campus Cartuja, s/n. \\ 18071 Granada, España \\ mpinto@ugr.es
}

\section{Resumen}

Se analiza dentro del contexto internacional la evolución temática de la investigación sobre Información y Documentación en español entre 1978 y 2019. Para ello se recuperaron las referencias bibliográficas relevantes de la base de datos LISA (Library and information science abstracts); y sus títulos y resúmenes fueron tratados con el método Latent Dirichlet Allocation ( $L D A)$, una técnica estadística de modelado temático. Se hallaron 19 conjuntos temáticos que fueron analizados, etiquetados y sistematizados en cuatro grandes áreas: procesos, tecnologías de la información, bibliotecas y documentaciones especializadas. Seguidamente se compararon los resultados en español con los resultados obtenidos anteriormente con la misma metodología y sobre la misma fuente a nivel internacional. En conclusión, la bibliografía LIS en español sigue en su conjunto las tendencias internacionales: el foco temático de la investigación ha variado en los últimos 50 años desde las bibliotecas y las organizaciones informacionales a los usuarios y el desarrollo de sistemas y soluciones específicas. Sin embargo, presenta también ciertas características propias: la importancia de la investigación bibliométrica y en documentación biomédica; el interés sostenido por la investigación netamente bibliotecaria; y un cierto retraso en el abordaje de los aspectos tecnológicos, legales y educativos. Aunque la selección de artículos de LISA depende de decisiones editoriales, la aplicación del LDA a la bibliografía en español ha ofrecido resultados consistentes con el estudio de tendencias globales internacionales, estudios sobre otras fuentes semejantes y las observaciones de investigaciones anteriores. 


\title{
Palabras clave
}

Información y Documentación; Biblioteconomía y Documentación; LISA; LDA; Modelado temático; Evolución; Temas de investigación; Frentes de investigación; Tendencias de investigación; Estudios bibliométricos; España.

\begin{abstract}
The thematic evolution of LIS research in Spanish between 1978 and 2019 is analyzed within the international context. To this end, relevant bibliographic references were retrieved from the Library and Information Science Abstracts (LISA) database, and their titles and abstracts were treated using the latent Dirichlet allocation (LDA) method, a statistical thematic modeling technique. Nineteen thematic sets were found and analyzed, labeled, and systematized in four main areas: processes, information technologies, libraries, and specialized documentations. Next, the results in Spanish were compared with international results obtained previously using the same methodology. In conclusion, LIS literature in Spanish mainly follows the international trends: during the last 50 years, the thematic focus of research has shifted from libraries and informational organizations to users and the development of specific systems and solutions. However, LIS research in Spanish also presents distinct characteristics: the importance of bibliometric research and biomedical documentation; research in the library area; and a certain delay in addressing technological, legal, and educational aspects. Although the selection of LISA articles depends on editorial decisions, the application of $L D A$ to the peer-reviewed literature in Spanish provided results that are consistent with the international global trends, studies on other similar sources, and overall the state of the art.
\end{abstract}

\section{Keywords}

Library and Information Science; LIS; LISA; LDA; Topic modeling; Evolution; Research topics; Research fronts; Research trends; Bibliometric studies; Spain.

\section{Financiación}

Este artículo se ha realizado en el marco de los proyectos CSO2015-65448-R (Mineco/Feder), PGC2018-093755B-I00 (MCIU) y CSO2016-80147-R (Mineco/Feder), sin cuyo patrocinio no hubiera sido posible.

Agradecimientos

Agradecemos las sugerencias a los revisores anónimos, que han servido para mejorar este trabajo.

\section{Introducción}

Este artículo se inscribe dentro del conjunto de trabajos que intentan describir la evolución temática de la producción científica en LIS de una forma global, por contraposición a los estudios más centrados en identificar los temas centrales de los investigadores más citados - los frentes de investigación-, de los que puede ser prototípico el estudio de Åström (2007), aunque los resultados son lógicamente compatibles y complementarios. La investigación bibliométrica sobre la evolución de LIS en español ha sido importante y aborda también cuestiones no temáticas como los rankings y redes de autorías, centros, grupos de investigación y revistas, o el análisis de aspectos transversales como las metodologías de investigación, que no se van a tratar aquí, pero que cuentan con buenas revisiones recientes (Guallar et al., 2017; Ferran-Ferrer et al., 2017).

A partir de los datos e informaciones obtenidas en investigaciones anteriores (Figuerola; García-Marco; Pinto, 2017) sobre la evolución de las áreas temáticas en la investigación científica en LIS, este trabajo intenta particularizar la visión de la investigación realizada en español en este campo, buscando profundizar en las diferencias y semejanzas con la evolución internacional; y, especialmente, intentando explicar tales diferencias y semejanzas.

En este trabajo se consideran sinónimos y se utilizan indistintamente los términos Biblioteconomía y Documentación, Información y Documentación, Ciencia de la Información y las Bibliotecas, y Ciencias de la Documentación, todos ellos usados por comunidades de investigadores hispanohablantes. El tercero es la traducción literal del término como se denomina al campo de investigación y actividad profesional en el ámbito anglosajón e internacional, Library and Information Science (LIS), cuyas siglas se utilizarán también para evitar reiteraciones y por ser mucho más conocidas que las españolas (CIB), apenas usadas. El primero (Biblioteconomía y Documentación) se utilizó durante el periodo inicial de los estudios universitarios en nuestro país, y todavía sigue siendo usado en muchos trabajos de investigación; y el segundo (Información y Documentación) ha tenido más aceptación en este siglo (García-Marco, 2013). La publicación del Libro blanco del grado en información y documentación por parte de la Agencia Nacional de Evaluación de la Calidad y Acreditación $(A n e c a, 2004)$ puede considerarse un pivote entre estas dos épocas marcadas por la terminología. Otra denominación popular en nuestro país ha sido Ciencias de la Documentación.

Es cierto que las diferentes denominaciones suponen concepciones diferentes de las relaciones entre las subdisciplinas y su importancia relativa, como ha explicado López-Yepes (2015, pp. 67-99), pero sirven para describir sus límites de forma casi equivalente, dentro de una cierta indefinición tradicional en el campo de investigación (Saracevic, 1999; Delgado-López-Cózar, 2002; López-Yepes, 2015; Hjørland, 2018a,b). 
Este trabajo está organizado así: en la sección siguiente se presentan algunos trabajos existentes sobre la definición de áreas temáticas en LIS, con incidencia específica en la producción científica en español; a continuación se expone la metodología seguida. Después, se presentan y discuten los resultados obtenidos, primero en una visión de conjunto y después profundizando en las cuatro grandes áreas temáticas encontradas. Finalmente, se ofrecen unas conclusiones y la bibliografía utilizada.

\section{Trabajos previos}

El análisis de la evolución temática de LIS cuenta con una amplia tradición científica, tanto dentro de estudios bibliométricos de amplio espectro como más específicos. Destaca como arranque de mucha de la investigación moderna la clasificación de Järvelin y Vakkari $(1990,1993)$ que ha seguido siendo usada a efectos comparativos en varios estudios semejantes a pesar de que los propios autores manifiestan sus limitaciones (Tuomaala; Järvelin; Vakkari, 2014; Hjørland, 2018a,b).

Dicha clasificación es un esquema detallado que divide LIS en once campos:

- The professions in library and information science (LIS) services,

- Library history,

- Publishing (including book history),

- Education in LIS,

- Methodology,

- Analysis of LIS,

- Library and information-service (L\&I) activities,

- Information storage and retrieval (ISR),

- Information seeking,

- Scientific and professional communication,

- Other aspects of LIS.

Esa clasificación ha sido también utilizada en importantes estudios sobre la evolución temática de la disciplina en español (Jiménez-Contreras; De-Moya-Anegón, 1997; Cano, 1999; Delgado-López-Cózar, 2000; 2002) y ha sido citada por muchos otros.

Con una estrategia en dos niveles muy semejante a la seguida en este trabajo, Blessinger y sus colaboradores (Blessinger; Frasier, 2007; Blessinger; Hrycaj, 2010) desarrollaron una clasificación de 43 temas agrupados en cinco categorías generales:

- library operations,

- library/information science profession,

- publishing/publishing studies,

- research in librarianship/users,

- technology.

Sin embargo, estos autores no buscaron los temas estadísticamente, sino a partir de los descriptores de las bases de datos evaluadas.

Con este último enfoque, varios investigadores españoles y extranjeros también han desarrollado clasificaciones temáticas propias específicamente orientadas al caso español. Así, Ríos-Hilario (2001) indica la presencia de tres grandes bloques -documentos, centros y usuarios-y plantea una clasificación de temas:

- generalidades sobre las Ciencias de la Información,

- organismos de documentación,

- fuentes documentales,

- sistemas y aplicaciones,

- análisis de la información,

- almacenamiento,

- reproducción y difusión, y

- apoyo a la información.

En una revisión posterior, insiste en la necesidad de adecuar la clasificación a la rápida evolución de los temas de investigación (Ríos-Hilario; Travieso Rodríguez, 2013). Por su parte, Kawalec (2013) utiliza el JITA Classification Schema of Library and Information Science, del repositorio e-LIS (E-prints in Library and Information Science).

Finalmente, Ferran-Ferrer et al. (2017) han elaborado también recientemente una clasificación empírica (tabla 1) partiendo de descriptores del Tesauro de biblioteconomía y documentación (Mochón-Bezares; Sorli-Rojo, 2002). 
Tabla 1. Lista de temas en el estudio de Ferran-Ferrer et al.(2017)

\begin{tabular}{|c|c|c|c|c|c|c|}
\hline \multirow{2}{*}{ Categoría temática } & \multicolumn{2}{|c|}{ Total } & \multicolumn{2}{|c|}{ Investigación } & \multicolumn{2}{|c|}{ No investigación } \\
\hline & $\mathbf{N}$ & $\%$ & $\mathbf{N}$ & $\%$ & $\mathbf{N}$ & $\%$ \\
\hline Fuentes y recursos de información & 86 & 14,80 & 57 & 14,43 & 29 & 15,59 \\
\hline Estudios métricos & 79 & 13,60 & 75 & 18,99 & 4 & 2,15 \\
\hline Tecnologías de información & 71 & 12,22 & 36 & 9,11 & 35 & 18,82 \\
\hline Técnicas adicionales y otras disciplinas & 67 & 11,53 & 47 & 11,90 & 20 & 10,75 \\
\hline Comunicación & 54 & 9,29 & 40 & 10,13 & 14 & 7,53 \\
\hline Principios teóricos y aspectos generales & 45 & 7,75 & 38 & 9,62 & 7 & 3,76 \\
\hline Unidades y servicios de información & 39 & 6,71 & 12 & 3,04 & 27 & 14,52 \\
\hline Profesionales & 38 & 6,54 & 24 & 6,08 & 14 & 7,53 \\
\hline Acceso y recuperación de información & 31 & 5,34 & 17 & 4,30 & 14 & 7,53 \\
\hline Proceso técnico & 30 & 5,16 & 16 & 4,05 & 14 & 7,53 \\
\hline Usuarios & 28 & 4,82 & 25 & 6,33 & 3 & 1,61 \\
\hline Archivística & 13 & 2,24 & 8 & 2,03 & 5 & 2,69 \\
\hline
\end{tabular}

Otros trabajos sobre la distribución y evolución temática de la investigación española en LIS con otras metodologías como coocurrencia de palabras clave y redes de citación pueden consultarse en Olmeda-Gómez, Ovalle-Perandones y Perianes-Rodríguez (2017).

\section{Metodología}

\subsection{LISA como fuente de información}

La fuente de información utilizada en este trabajo ha sido LISA (Library and Information Science Abstracts), una base de datos bien conocida y utilizada en el mundo de la Ciencia de la Información desde hace años. LISA fue creada en 1969 a partir de los resúmenes que publicaba la Library Association del Reino Unido tras un acuerdo de colaboración con Aslib para incluir registros de lo que entonces era un campo en rápido crecimiento (Gilchrist; Presanis, 1971; Gilchrist, 1969). Desde entonces, y con varias fusiones e incorporaciones por medio (Searing, 2012), LISA ha llegado a nuestros días. Distribuida a través de ProQuest, indiza y resume 487 revistas de 45 países en 20 idiomas, siempre dentro del campo de la biblioteconomía, documentación, edición y tecnología de la información. Se actualiza con la adición de alrededor de 1.000 registros por mes (ProQuest, 2016).

El 24 de enero de 2020 se descargaron de LISA todos los registros cuyo campo LA (lengua) = Spanish . Resultó un total de 7,455 registros desde 1969 (fecha de comienzo de LISA) hasta 2019 (probablemente, todavía en curso) de 192 fuentes. Las fuentes en español solo se hacen presentes de forma notable a partir de 1978, aumentando la pendiente a partir de 1993 y duplicándose entre 2006 y 2017 (figura 1). El crecimiento de las publicaciones ha sido, por tanto, muy intenso. Es importante señalar que los totales no son fiables para los dos últimos años, pues se aprecia que varias fuentes se encuentran

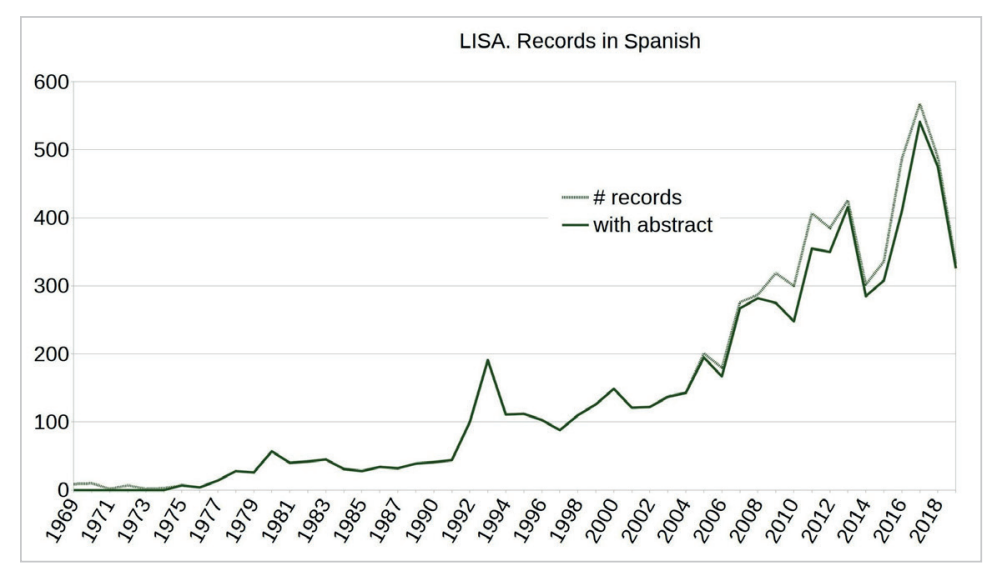

Figura 1. Crecimiento de la cobertura de LISA en español

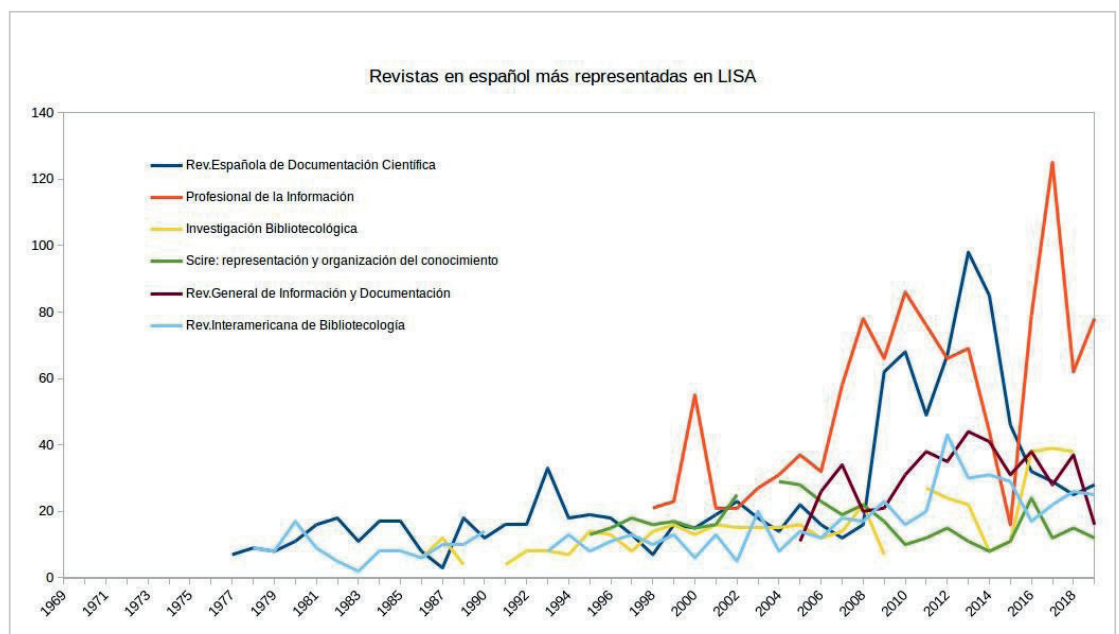

Figura 2. Representatividad de las principales revistas en español recogidas en LISA 
en proceso de indización, lo que se refleja en un acusado descenso de volumen en 2018 y 2019.

La tabla 2 muestra la distribución de registros por fuente, el total a la izquierda y los revisados por pares a la derecha. A pesar de que LISA los cataloga como documentos en español, algunas fuentes están en portugués y otras en catalán. Aunque todos los registros están clasificados por LISA como artículos de revista, en realidad hay también comunicaciones a congresos, informes, capítulos de libro, noticias, reseñas, etc.

Comparando las columnas primera y tercera de la Tabla 2 se constata que la información que proporciona LISA sobre la revisión por pares no siempre es precisa. En efecto, numerosas revistas en español que sí revisan por pares, sin embargo, figuran en LISA como no revisadas. Por esta razón se decidió trabajar sobre el conjunto de los registros, sin limitarse a los que vienen marcados como revisados por pares, que, como se puede apreciar, constituyen además una muestra muy limitada en tamaño.

Otro sesgo importante de LISA es la selección editorial. LISA no vacía sistemáticamente las fuentes, sino que los incluye en su base de datos en la medida en que se ajusta a sus intereses o procesos internos (figura 2).

Esta situación no es única. En mucha mayor medida que las bases de datos construidas a partir del análisis cuantitativo de citas, que seleccionan a partir de modelos bibliométricos, las bases de datos como LISA están históricamente afectadas por las políticas editoriales de selección. Esto se ha apreciado en investigaciones similares realizadas sobre otras bases de datos, en particular en los estudios de Prebor (2007; 2010) sobre las tesis de ProQuest digital dissertations clasificadas en los campos de 'Library Science' e 'Information Science'. Significativamente, el segundo estudio sobre la interdisciplinariedad de la investigación en LIS (Prebor, 2010) ofrece campos temáticos sorprendentemente semejantes a los de este estudio. Este hecho avala la representatividad de la selección realizada por los editores de estas bases de datos especializadas, a pesar de su carácter limitado. Sin embargo, este sesgo debe ser tenido en cuenta y, por ello, la evolución temática de la investigación sobre la disciplina en España tal y como se presenta en este trabajo debe considerarse en función del punto de vista de los editores de LISA y de la selección de fuentes y artículos realizada por ellos. Desde un punto de vista estadístico, esta selección de fuentes sobre un conjunto de documentos menor produce también unos gráficos con mayor dispersión, mucho más aserrados, que dificultan la percepción de las tendencias, razón por la que se ha calculado también la pendiente de regresión de las tendencias a efectos de comparación con el estudio internacional.

La bibliografía en español ocupa la sexta posición en la producción recogida en LISA (figura 3). La producción específicamente española se sitúa en la posición undécima, una posición compatible con la que ocupa en la producción científica internacional, aunque el número de artículos quede muy por debajo de su dimensión real, lo que es comprensible dado el origen y el enfoque geográfico de la base de datos. Estos datos, sin embargo, son coherentes con el diagnóstico realizado por Ferran-Ferrer et al. (2017) a partir de Web of Science y Scopus de que
Tabla 2. Revistas en español recogidas en LISA por número de registros (1969-2019)

\begin{tabular}{|c|c|c|}
\hline $\begin{array}{l}N^{\circ} \text { de } \\
\text { artículos }\end{array}$ & $\begin{array}{l}\text { Revisados } \\
\text { por pares }\end{array}$ & Fuentes \\
\hline 1.677 & 517 & Revista española de documentación científica \\
\hline 1.170 & & Profesional de la información \\
\hline 466 & 195 & Investigación bibliotecológica \\
\hline 458 & & Scire: Representación y organización del conocimiento \\
\hline 451 & 11 & Revista general de información y documentación \\
\hline 403 & & Revista interamericana de bibliotecología \\
\hline 324 & 1 & Documentación de las ciencias de la información \\
\hline 314 & & Bibliotecas. Anales de investigación \\
\hline 261 & & Anuario ThinkEPI \\
\hline 243 & & Ibersid: Revista de sistemas de información y documentación \\
\hline 236 & & Anales de documentación \\
\hline 230 & 94 & Ciencias de la información \\
\hline 203 & & Ciência da informação \\
\hline 192 & 31 & Información cultura y sociedad \\
\hline 174 & & Revista interamericana de bibliotecología \\
\hline 174 & & Informação \& informação \\
\hline 138 & & Boletín de la Asociación Andaluza de Bibliotecarios \\
\hline 138 & 4 & Análisis de tendencias en información y documentación \\
\hline 133 & & Anales de documentación \\
\hline 111 & & $\begin{array}{l}\text { Enl@ce: Revista venezolana de información, tecnología y } \\
\text { conocimiento }\end{array}$ \\
\hline 106 & & Revista Aibda \\
\hline 64 & & Boletín de la Anabad \\
\hline 55 & & @tic \\
\hline 38 & & Ítem \\
\hline 33 & & BiD. Textos universitaris de biblioteconomia i documentació \\
\hline 29 & & $\begin{array}{l}\text { Proceedings of the Eighth Andalucian libraries assembly, } \\
\text { Huelva, Spain, 12-14 May } 94\end{array}$ \\
\hline 27 & 20 & Information research \\
\hline 20 & & $\begin{array}{l}\text { Proceedings of the first ISKO-Spain conference, Madrid, 4-5 } \\
\text { Nov } 93\end{array}$ \\
\hline 18 & & Informação \& sociedade \\
\hline 18 & & Dirección General de Archivos y Bibliotecas Boletín \\
\hline 14 & & Revista latinoamericana de documentación \\
\hline 13 & & Ekare \\
\hline 13 & & $\begin{array}{l}\text { Aibda. Asociación Interamericana de Bibliotecarios y Docu- } \\
\text { mentalistas Agricolas, 1980, 69-89. ss }\end{array}$ \\
\hline 11 & & Perspectivas em gestão \& conhecimento \\
\hline
\end{tabular}


"el número de los artículos de investigación publicados [...] es comparable con el número de trabajos publicados internacionalmente".

\subsection{Técnicas de modelado de} temas

Este trabajo se ha realizado con una metodología muy específica conocida como modelado de temas. Por modelado de temas se conoce una serie de técnicas que permiten analizar de manera automática los temas tratados en los documentos que conforman una colección; esto incluye la detección de dichos temas, su intensidad y su vinculación con otra información adicional disponible sobre los documentos; por ejemplo, el idioma o la fecha.

Una de las técnicas más difundidas es la conocida como Latent Dirichlet Allocation (LDA) (Blei et al., 2003), que parte de la idea de que en una colección de documentos existe un conjunto de temas; y cada documento individual puede ser considerado una mezcla, en diferentes proporciones, de esos temas. La proporción de un tema específico en un documento determinado puede ser incluso de 0 , es decir, no estar presente en absoluto. Un caso habitual es encontrar documentos que contengan una elevada proporción de un tema determinado (conocido a veces como top topic) y proporciones muy bajas de los demás (Maskeri et al., 2008).

La misión del modelado de temas consiste, pues, en identificar el conjunto de temas de la colección documental y en establecer la proporción de cada tema en cada documento. Estas operaciones se basan en la coocurrencia de palabras en los mismos documentos; y permiten establecer conjuntos de palabras definitorias, con mayor o menor peso, de cada tema. La presencia de unas u otras palabras en cada documento permite también estimar el porcentaje o proporción que cada tema juega en el contenido de ese documento (Blei, 2012). Existen, de otro lado, varias implementaciones de LDA, lo que facilita su aplicación. Una de las más conocidas es Mallet (MacCallum, 2002), que viene en forma de librería y también como programa autónomo listo para ser usado; ésta es, precisamente, la implementación que se ha utilizado en el presente trabajo.

Se efectuó una descarga masiva de registros fechados entre 1978 y 2014. El conjunto de los 92.705 registros descargados, procesado mediante técnicas de topic modeling, fue la base de un estudio que permitió trazar las grandes áreas de investigación en LIS y su evolución a lo largo de tiempo (Figuerola; García-Marco; Pinto, 2017). El subconjunto de los registros escritos en español, puesto en relación con el resto de los registros, es la fuente que se ha utilizado para efectuar el análisis de la investigación en español en LIS.

LISA ofrece 22 campos, algunos repetibles (por ejemplo, autor y palabra clave). De ellos nos interesan los que informan sobre el idioma, obviamente, y los que contienen el título y el resumen en inglés. Con el título y el resumen en inglés de cada registro se constituyó un conjunto de documentos que fue sometido a un proceso de LDA a través de Mallet. Tan sólo se aplicaron algunas operaciones de análisis léxico ligero: normalización de caracteres y eliminación de una lista estándar de palabras vacías para el idioma inglés.

\subsection{Identificación empírica de los conjuntos temáticos}

La determinación del número de temas es un asunto controvertido (Arum et al., 2010) que requiere una cuidadosa valoración por expertos, que, en este caso, se realizó mediante un proceso de consenso Delphi, esto es, en sucesivas aproximaciones, por parte de los tres coautores. Trabajando sobre los conjuntos empíricos proporcionados por el modelo LDA, se les asignaron descriptores que categorizaran cada uno de ellos. A partir de los descriptores, cada experto propuso una rúbrica única que denominara clara e inequívocamente a cada conjunto. El análisis realizado en enero de 2020 para la bibliografía en español recogida en LISA ofreció 24 grupos empíricos, frente a los 19 identificados en el estudio internacional (Figuerola; García-Marco; Pinto, 2017).

Con una metodología semejante, pero ajustada a los resultados, dichos temas fueron a su vez clasificados en cinco grandes áreas a partir del juicio experto, para generar dos niveles de análisis:

- procesos,

- tecnologías de la información,

- biblioteconomía,

- documentaciones especializadas, e

- informetría.

Esta última macroárea es específica de la bibliografía en español en razón a su relevancia numérica, de la misma manera que en el estudio de 2017 se desagregaron las bibliotecas y las documentaciones especializadas para respetar la 
representatividad numérica de cada una a lo largo del periodo estudiado. Con todo, las tres últimas macroáreas pueden considerarse en su conjunto 'áreas de aplicación', como se puede apreciar en la tabla 3.

En la tabla 3 se señalan en rojo los temas obtenidos en el estudio de 2017 (global) que no tienen correspondencia en el actual (en español); y en azul los conjuntos que han surgido nuevos.

Tabla 3. Grandes áreas y temas de investigación detectados en LISA

\begin{tabular}{|c|c|c|}
\hline & Áreas & Temas \\
\hline \multirow{7}{*}{ Procesos } & \multirow{7}{*}{ Procesos transversales } & Teoría y epistemología \\
\hline & & Educación y profesionalización \\
\hline & & Desarrollo social \\
\hline & & Comportamiento informacional \\
\hline & & Derecho y ética de la información \\
\hline & & Preservación documental \\
\hline & & Organización del conocimiento \\
\hline \multirow{5}{*}{ Tecnologías } & \multirow{5}{*}{ Tecnologías } & Redes de comunicación \\
\hline & & Estadística avanzada \\
\hline & & Cambio tecnológico \\
\hline & & Representación y procesamiento automáticos de la información \\
\hline & & Servicios de búsqueda en línea \\
\hline \multirow{17}{*}{ Áreas de aplicación } & \multirow{4}{*}{ Biblioteconomía } & Aspectos políticos y sociales \\
\hline & & Gestión de bibliotecas y sus servicios \\
\hline & & Servicios de referencia \\
\hline & & $\begin{array}{l}\text { Catalogación y cooperación bibliotecaria } \\
\text { (bibliotecas y repositorios digitales) }\end{array}$ \\
\hline & \multirow{9}{*}{ Documentaciones especializadas } & Fuentes humanísticas \\
\hline & & Informetría (a sección aparte) \\
\hline & & Información y documentación sanitaria \\
\hline & & Información y documentación mediática \\
\hline & & Redes sociales \\
\hline & & Información y documentación educativa \\
\hline & & Información y documentación empresarial \\
\hline & & Gestión del conocimiento \\
\hline & & Gestión del conocimiento en educación superior \\
\hline & \multirow{4}{*}{ Informetría } & Metodologías y técnicas \\
\hline & & Estudios de citas e impacto \\
\hline & & Evaluación de la investigación \\
\hline & & Informetría aplicada (interdisciplinar) \\
\hline
\end{tabular}

A continuación, se describen con detalle:

El área "Procesos transversales" clasifica 7 campos temáticos que constituyen subdisciplinas transversales de las ciencias de la información y las bibliotecas, a diferencia de las otras cuatro áreas, que son o bien disciplinas auxiliares (tecnologías) fuera del campo estricto de la Biblioteconomía y la Documentación, o sobre todo especialidades temáticas: biblioteconomía como la más distintiva y diferentes documentaciones especializadas o transdiciplinas en las que LIS juega un papel importante (gestión del conocimiento). Los conjuntos temáticos de esta macroárea son los siguientes:

- "Teoría y epistemología" incluye artículos que se ocupan de los aspectos teóricos de la Biblioteconomía y la Documentación, su relación con los conceptos de información, conocimiento, comunicación, cultura, etc.

- La categoría "Educación y profesionalización” agrupa artículos sobre la educación, formación y desarrollo profesional de los practicantes e investigadores en Biblioteconomía y Documentación. Los artículos abordan tanto la formación académica en sus diferentes niveles (graduado, posgraduado y doctoral) como la educación profesional y continua proporcionada por las asociaciones, las empresas y las administraciones. También aparecen en este grupo artículos sobre los movimientos asociativos de los profesionales y académicos de la Información y la Documentación; y los estudios de análisis y prospección del mercado laboral y de los nichos de ocupación consolidados, emergentes o en decadencia.

- "Desarrollo social" etiqueta el conjunto de artículos que abordan la participación de los profesionales y científicos de la 
información en el desarrollo social, económico y cultural en los niveles internacional, nacional, regional o comunitario; y tanto los referidos a la población en general como a sectores específicos y, especialmente, desfavorecidos. El análisis LDA lo ha diferenciado de otro grupo muy relacionado, que se considera en el área de aplicaciones: la alfabetización informacional, que ha aparecido principalmente agrupada en la categoría "Educación y aprendizaje". En general, la Información y Documentación se ha percibido tradicionalmente como un actor clave en la promoción del desarrollo, y ese compromiso constante se manifiesta nuevamente en el caso de la Agenda 2030 para el Desarrollo Sostenible de la Organización de las Naciones Unidas y sus 17 objetivos de desarrollo sostenible (Singh, 2016; Chowdhury; Koya, 2017).

- La categoría "Comportamiento informacional" incluye investigaciones cuantitativas y cualitativas, así como reflexiones sobre el uso de fuentes, servicios y sistemas de información por los usuarios. Se corresponde con la especialidad de la Biblioteconomía y Documentación que tradicionalmente se ha denominado "estudios de usuarios" y que contemporáneamente es conocida como comportamiento informacional (information behaviour, en inglés).

- "Derecho y ética de la información" agrupa artículos relacionados con la manera correcta de adquirir, gestionar, comunicar y usar la información de acuerdo a los estándares éticos y las normas legales. Se trata de un campo muy amplio que abarca desde los derechos humanos básicos relacionados con la información (derecho a la información, privacidad...), derechos y deberes establecidos por el marco político y regulaciones gubernamentales específicas, como los de protección intelectual o transparencia; así como problemas éticos que, sin estar recogidos en el marco legal de forma específica, son objeto del debate social.

- "Preservación documental" agrupa el conjunto de artículos que se ocupa de la conservación de los documentos a largo plazo, tanto de los documentos históricos como de los actuales. Se aborda tanto desde un punto de vista general como especializado, según condiciones o tipos de formatos, lo que, actualmente, incluye como tema de la máxima actualidad la preservación de los documentos y de la información digitales. Incluye buena parte de los documentos relacionados con el proceso técnico en archivos.

- "Organización del conocimiento" incluye artículos sobre lenguajes documentales (clasificaciones, tesauros, encabezamientos de materia, taxonomías, folksonomías, etc.) y su utilización para la representación temática de los documentos a través de la indización y la reflexión teórica sobre estas herramientas y procesos, y sus contextos.

El área "Procesos" es contemplado en algún trabajo internacional sobre la evolución de la investigación en LIS (Milojevic et al., 2011), y también lo son algunos de sus temas específicos, como las cuestiones sociales (Janssens et al., 2006) o la formación y desarrollo profesional (Blessinger; Hrycaj, 2010).

El área "Tecnologías" reúne tres conjuntos de artículos que responden a la incorporación a la información y la documentación de principios, modelos y técnicas desde otras disciplinas científicas que han tenido un gran impacto en nuestra época. Todas ellas forman parte de una manera u otra del campo de las tecnologías de la información y la comunicación. Estos artículos realizan fundamentalmente operaciones de incorporación interdisciplinar. Sus conjuntos temáticos son los siguientes:

- "Cambio tecnológico" se corresponde con un conjunto de artículos que abordan la incorporación de la revolución de las tecnologías de la información y la comunicación en el campo de la Biblioteconomía y la Documentación. Incorporaría el conjunto detectado en el estudio de 2017 con la etiqueta "Redes de comunicación" (Figuerola; García-Marco; Pinto, 2017). Este grupo tiene una conexión estrecha con una parte importante del derecho de la información.

- “Representación y procesamiento automático de la información” reúne a artículos que tratan la automatización del procesamiento de la información. Puede ser mediante técnicas basadas en el procesamiento automatizado del lenguaje: metadatos, ontologías, procesamiento lógico..., que, en general, abordan el potencial semántico de la codificación lingüística, haciendo su semántica explícita mediante diferentes técnicas, tradicionalmente relacionadas con la investigación en sistemas expertos y la inteligencia artificial. Y en el caso de estudio también mediante procedimientos estadísticos avanzados. Este grupo presenta una fuerte conexión con el de "Organización del conocimiento".

- "Servicios de búsqueda en línea" constituye el conjunto de artículos que se ocupan del diseño, evaluación y mejora de servicios de búsqueda de información en red: interfaces de búsqueda en bases de datos; bibliotecas digitales; catálogos de acceso público online (opac); museos digitales; portales de internet; navegación, organización y búsqueda en sitios web; sistemas de gestión de documentos; descubrimiento de fuentes de información; etc.

Las aplicaciones estadísticas e informáticas a la Biblioteconomía y la Documentación también se reconocen como un área fundamental en varios de los estudios bibliométricos (Åström, 2007; Blessinger; Frasier, 2007; Janssens et al., 2006). Jiménez-Contreras, Delgado-López-Cózar y Ruiz-Pérez (2006, pp. 382-383) han constatado la importancia también para el caso español de "los especiales lazos entre la ByD y las ciencias de la computación".

La tercera área la constituye la especialidad más sólida de las Ciencias de la Documentación: la Biblioteconomía. Las bibliotecas han sido consideradas uno de los campos nucleares en numerosos estudios teóricos (Webber, 2003; Bates; Maack, 2010) y bibliométricos (Blessinger; Frassier, 2007; Blessinger; Hrycaj, 2010; Koufogiannakis; Slater; Crumley, 2004; Milojevic et al., 2011), algunos incluso la consideran una disciplina separada de la Ciencia de la Información (Saracevic, 1999). Los temas componentes se han agrupado en tres grandes conjuntos empíricos. El primero es más general, y los otros dos han quedado desgajados de él por su importancia numérica y la fuerte vinculación interna. Se trata de: 
- "Aspectos políticos y sociales" agrupa artículos que abordan el papel de las bibliotecas -nacionales, públicas y académicas fundamentalmente- y el rol de los bibliotecarios en la construcción de la comunidad política y social, a través de conceptos como la formación y la promoción de la lectura.

- "Gestión de bibliotecas" agrupa los artículos que tratan de la teoría de la gestión, diseño y evaluación de las bibliotecas y sus servicios. Como ocurre en otras secciones se trata tanto de trabajos de carácter general como especializado en diferentes tipos de bibliotecas (especiales, locales, nacionales, académicas, públicas, escolares...). El algoritmo LDA ha desagregado automáticamente dos grandes grupos que suelen considerarse dentro de la gestión de bibliotecas, pero que, por la estrecha relación de sus temas, han terminado formando conjuntos independientes. Se trata del servicio de referencia y de la función de catalogación, que se consideran a continuación:

- "Catalogación y cooperación bibliotecaria" da título a un conjunto de documentos que tratan sobre la catalogación bibliográfica y todo el contexto que la rodea. Aunque el conjunto pueda parecer a primera vista algo artificial, tiene todo el sentido si se piensa que durante gran parte de la historia contemporánea de las bibliotecas, la catalogación bibliográfica y el desarrollo de normas y formatos de catalogación e intercambio han sido el centro de las actividades de cooperación entre las bibliotecas y han constituido la base para otros servicios como el intercambio de documentos y la creación de catálogos colectivos, por lo que han sido centrales en el origen de organizaciones como la IFLA o la OCLC. Como resultado de la aplicación del algoritmo, las cuestiones más relacionadas con la búsqueda, notablemente los opacs, quedaron en otro tema, a saber: "Servicios de búsqueda en línea". En el caso de la bibliografía en español, el conjunto resultante tiene especial relación con las bibliotecas y repositorios digitales, con un neto componente de innovación tecnológica.

Debe notarse, por último, la desaparición de un grupo específico de "Servicios de referencia”, que sí aparecía en el estudio de 2017 para la bibliografía LIS internacional en LISA (Figuerola; García-Marco; Pinto, 2017).

La cuarta área incorpora diversas documentaciones especializadas, que han llevado su curso de forma autónoma respecto a la Biblioteconomía, especialmente por abordar también cuestiones como sistemas de gestión de datos y documentos o plataformas y sitios web idiosincrásicos. Está formada por ocho especialidades:

- "Fuentes humanísticas" encabeza un grupo de artículos aparentemente muy diverso, pero que tienen en común la utilización de fuentes bibliográficas o documentales históricas, literarias, culturales, artísticas..., en la investigación humanística, en el marco de investigaciones realizadas en archivos y bibliotecas, y requiriendo un conocimiento muy especializado de sus fundamentos y técnicas. Aunque llama la atención la ausencia de un conjunto distintivo de archivos, en esta categoría se pueden encontrar muchos estudios relacionados con su parte histórica.

- "Información y documentación sanitaria" intitula artículos sobre la aplicación de la Biblioteconomía y la Documentación al sector de la salud, tanto en su vertiente de investigación como práctica. Los artículos tratan los diferentes sectores de interés (investigadores biosanitarios, médicos y otros profesionales de la salud, pacientes y población en general o por sectores específicos) y, por lo general, en relación con las tecnologías de la información y los sistemas de información.

- "Información y documentación mediática" agrupa artículos sobre la gestión y el uso de la información y la documentación en los medios de comunicación de masas. Incluye tanto artículos muy aplicados y especializados en cuanto a las audiencias o medios, como otros generales y de espectro muy amplio.

- "Redes sociales" es un nuevo conjunto que ha aparecido, muy en relación con el anterior, y que refleja el gran interés que ha despertado entre la comunidad científica y profesional el fenómeno de los medios sociales, sus tecnologías de base y su impacto social y político.

- El tema "Información y documentación educativa" agrupa dos grandes sectores de artículos netamente diferenciados. Por un lado, destaca el desarrollo y la utilización de sistemas de información orientados al aprendizaje, las denominadas plataformas de aprendizaje. El otro sector es el que tradicionalmente se ha conocido como formación de usuarios y más recientemente como alfabetización informacional (alfin) o desarrollo de competencias informacionales, bien sea desde un punto de vista general o bien sectorial, por ejemplo, dirigido a escolares, estudiantes universitarios noveles, pequeños empresarios, inmigrantes, etc.

- La "Información y documentación empresarial” agrupa los sistemas de gestión de la información empresarial y su aplicación para mejorar el desempeño competitivo de las organizaciones en los mercados.

- La "Gestión del conocimiento" es un campo de investigación, ligado a las ciencias de la administración, que estudia la creación, gestión, intercambio y uso de la información organizacional, esté o no documentada. Su objetivo es, por un lado, que el conocimiento sea adecuadamente codificado, organizado y recuperado; y por el otro que la información almacenada se aproveche y utilice para la creación de nuevo conocimiento y para resolver los problemas que se van presentando. Se trata de un área de investigación interdisciplinar en la que participan científicos y profesionales de disciplinas tan diversas como la administración de empresas, los sistemas de información, la ciencia de la administración, la biblioteconomía y documentación o los recursos humanos, entre otras. El alto nivel de abstracción del concepto de "gestión del conocimiento" sobre las diferentes especialidades de la información y la documentación hizo pensar en su punto álgido que podría constituir un paradigma de integración del área LIS. Dichas esperanzas han quedado algo minimizadas con el paso del tiempo, y la gestión del conocimiento parece actualmente más ligada a las ciencias de la gestión. 
- El grupo de "Gestión del conocimiento en educación superior" es un grupo muy distintivo que aplica los conceptos del bloque anterior -gestión del conocimiento- a la mejora de funciones y procesos en las instituciones de educación superior. Es el conjunto temático, con todo, más difuso y de límites menos claros que ha surgido como resultado de la aplicación de la metodología.

En relación con la fuerte especialización de las documentaciones, Jiménez-Contreras, Delgado-López-Cózar y Ruiz-Pérez (2006, p. 376) han mostrado el carácter multidisciplinar, transversal e instrumental de la investigación en Información y Documentación: lo publicado fuera de la categoría Information Science and Library Science (ISLS) en WoS era ya entonces prácticamente el $50 \%$ de la producción española y un tercio de los autores trabajaban "en instituciones no ligadas directamente a la ByD". A diferencia de lo que ocurría a nivel internacional, no ha aparecido un conjunto distintivo dedicado a la "informacion y documentación empresarial" (business information).

La quinta área la constituyen los conjuntos temáticos sobre "Informetría” que, en el caso de la bibliografía en español, se han separado de la sección de documentaciones especializadas para formar una macroárea propia por su importancia. Como es sabido, esta disciplina aborda el estudio de la información desde un punto de vista cuantitativo, en su gran mayoría con objetivos bibliométricos y cienciométricos, esto es, buscando describir la evolución de las ciencias a partir de las publicaciones científicas, sobre todo revistas, y buscando leyes y modelos que la describan de forma predictiva y la expliquen. En el presente estudio se han hallado cuatro subconjuntos empíricos:

- "Metodologías y técnicas" son artículos bibliométicos en los que se priorizan los problemas metodológicos.

- "Estudios de citas e impacto" es el conjunto que ha agrupado los artículos que se centran en el análisis del impacto de autores, revistas, instituciones, países y áreas geográficas mediante el análisis de citas.

- "Evaluación de la investigación" es un grupo de artículos especialmente relacionada con la evaluación de la investigación de las instituciones y departamentos universitarios, una de las principales funciones de la universidad.

- "Informetría aplicada (interdisciplinar)" está formado por artículos que aplican técnicas informétricas a la resolución de problemas variados de evaluación de servicios.

La importancia del análisis de la comunicación científica y profesional (Järvelin; Vakkari, 1990, 1993; Jiménez-Contreras; De-Moya-Anegón, 1997; Cano, 1999; Delgado-López-Cózar, 2000; 2002), del análisis de la información (Ríos-Hilario, 2001) y de los estudios métricos (Ferran-Ferrer et al., 2017) ha sido remarcada por todos los especialistas que se han ocupado de la evolución temática de la investigación en LIS, tanto a nivel internacional como específicamente en español.

A continuación, se presentarán los resultados internacionales y por conjuntos temáticos, y se discutirán comparando la investigación en español recogida en LISA en estos treinta y seis años de historia de la disciplina con la producción internacional recogida en esa misma base de datos y analizada en un estudio anterior (Figuerola; García-Marco; Pinto, 2017).

La distribución de las grandes áreas en la bibliografía en español es diferente que en la que se halló para la producción internacional

\section{Resultados y discusión}

\subsection{Evolución global}

La distribución de las grandes áreas en la bibliografía en español es diferente que en la que se halló para la producción internacional (figura 4). El área de procesos transversales es mayor, con un grupo destacado de teoría y epistemología y otro menor de organización del conocimiento que no emergieron para el conjunto internacional. El área de tecnologías es señaladamente inferior en el caso de la bibliografía en español, dónde faltan grupos distintivos para redes de comunicación y estadística avanzada. Sin embargo, los de áreas de aplicación en su conjunto -sumando biblioteconomía, documentación especializadas y bibliometría- son muy semejantes. Destaca la gran importancia que tiene dentro de la investigación en español la informetría, a la que en este estudio ha habido que promover a macroárea por su importancia cuantitativa y relativa a las otras.

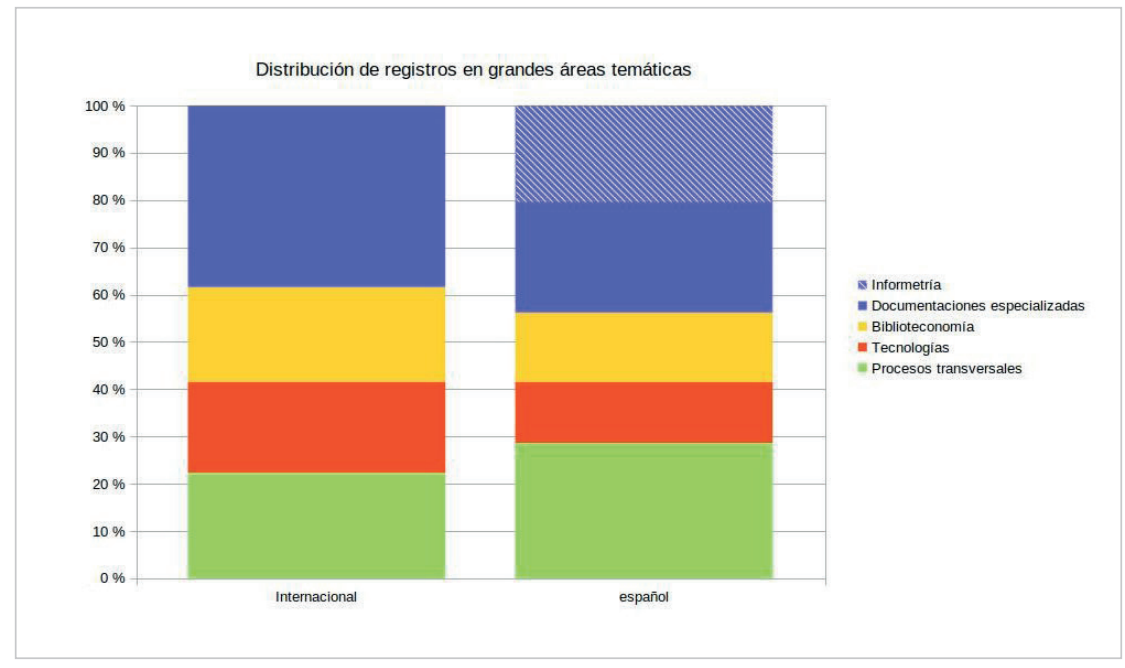

Figura 4. Comparación entre las grandes áreas en LISA: datos internacionales y artículos en español 
Comparando ambos gráficos, se aprecian tendencias comunes y divergencias. Respecto a las tendencias compartidas destaca el crecimiento de las documentaciones especializadas, incluyendo la informetría, y la pérdida de importancia de la biblioteconomía, muy reducida y más rezagada en la bibliografía en español. En cuanto a las divergencias, se aprecia el descenso del interés por el seguimiento de nuevas tecnologías desde 2012 y en las investigaciones que hemos denominado transversales y que tienen un enfoque más genérico.

Sin embargo, mientras que el gráfico por macroáreas temáticas de la evolución internacional (figura 5) es bastante claro, el gráfico para la investigación en español (figura 6) es más difícil de interpretar. Como se ha explicado anteriormente, esto se debe a que el número de documentos para el gráfico internacional es grande, mientras que para el español es más reducido y probablemente más afectado por las políticas de indización de LISA. Por ello, ofrece picos muy intensos y un gráfico con apariencia de dientes de sierra. Por ello, como se dijo anteriormente, para obviar este problema y obtener indicadores claros de tendencia, se ha optado por calcular las pendientes de las rectas de regresión, normalizándolas para hacerlas comparables, tanto para los periodos equivalentes (español 1975-2014, internacional 1978-2014) (figura 7), como para el total en español (español 1975-2019, internacional 1978-2014) (figura 8).

Utilizando las rectas de regresión, se observa que la investigación en español comparte en general las tendencias internacionales por grandes áreas, pero hay diferencias significativas. Es ligeramente decreciente en el caso del macrosector 'bibliotecas', con unas magnitudes muy semejantes y con apenas cambios en todo el tracto cronológico estudiado, aunque fuera del periodo de comparación se aprecia una recuperación de la investigación en español. En el caso de las tecnologías e informetría, las tendencias son positivas, esto es, la investigación se ha incrementado. Sin embargo, en lo que se refiere a procesos transversales se aprecia una fuerte disminución de la investigación en español, mientras que en el caso internacional permanece estable. Esto se debe a que la bibliografía de los años noventa tenía un carácter más general, ligado al despegue de los investigadores universitarios, mientras que en la actualidad la investigación se ha especializado mucho. También se observa que las documentaciones especializadas en español han 
perdido importancia relativa, aunque se han recuperado espectacularmente al final del periodo estudiado en este artículo.

Estos resultados son compatibles con el panorama que dibujan Jiménez-Contreras y De-Moya-Anegón (1997) y Cano (1999) para el frente de investigación universitario, que sintetiza Delgado-López-Cózar (2000; 2002) caracterizándolo por

"la falta de homogeneidad temática y la aproximación teórica a diferentes problemas de interés general en la disciplina, de una parte, $y$ el interés por los temas relacionado con la formación de los profesionales, de otra" (Delgado-López-Cózar, 2002, p. 131);

y que se confirma en un estudio posterior recoge gran parte del periodo investigado, en el que Olmeda-Gómez, Ovalle-Perandones y Perianes-Rodríguez (2017) confirman que

"la duración de los períodos de mayor intensidad para las frases nominales relacionadas con estos clusters es breve, lo que denota una baja estabilidad en la atención prestada a los temas formulados por la comunidad española de investigadores citantes" y que "[...] la mayor parte de la investigación no se encuentra dentro estructuras temáticas".

En lo que se refiere al área de tecnologías, la investigación española recogida en LISA le ha prestado una atención importante, pero menor, aunque las diferencias no son tan extremas. Como gran parte de la producción recogida en LISA es anglosajona (figura 3), esta diferencia se podría interpretar como un retraso en la adopción de tecnologías que han surgido principalmente en Estados Unidos y que han llegado primero al norte de Europa, aunque después de un interés inicial, la presencia de investigación española de este clúster no acompaña a la importancia recibida a nivel internacional. Da

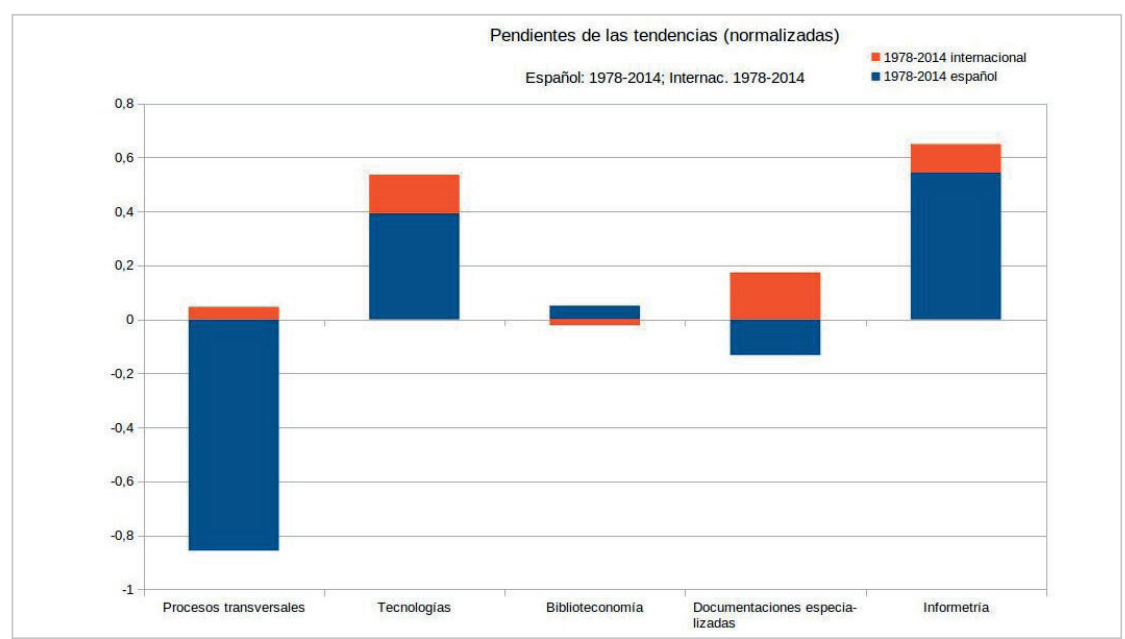

Figura 8. Comparación de las pendientes de las rectas de regresión de la bibliografía en español e internacional en LISA (español 1975-2014, internacional 1978-2014)

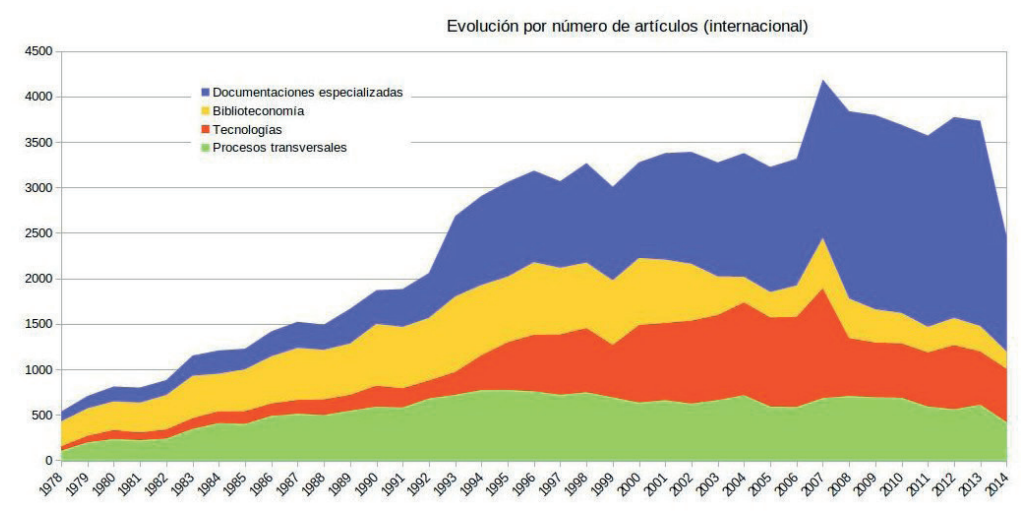

Figura 9. Evolución internacional en LISA del número de artículos por macroáreas temáticas

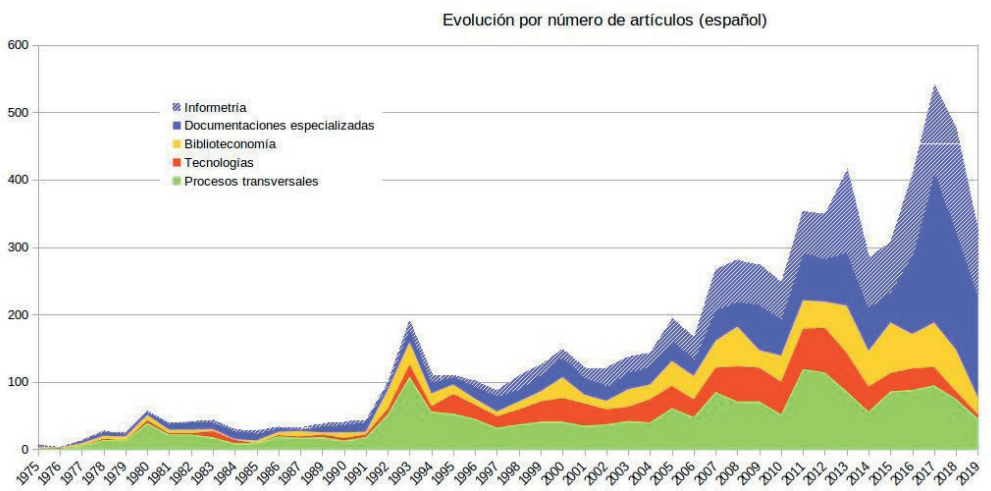

Figura 10. Evolución en LISA del número de artículos en español por macroáreas temáticas

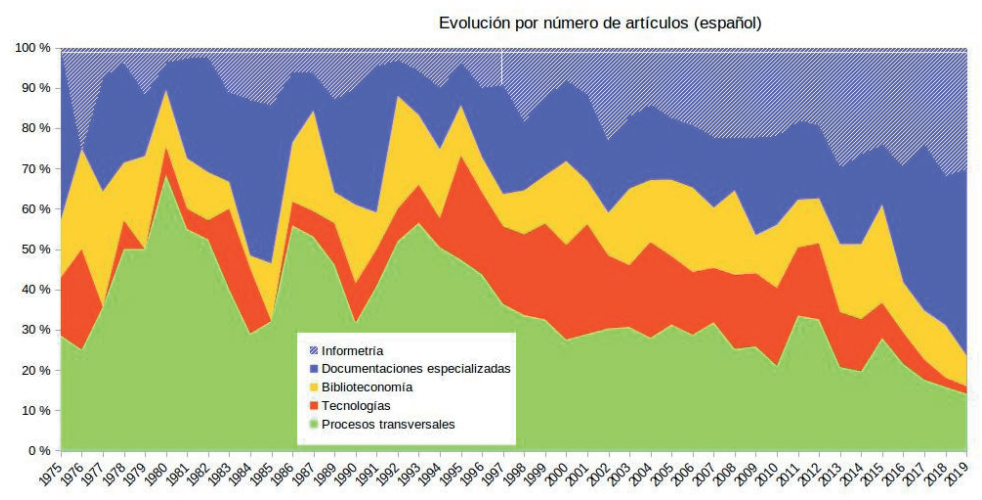

Figura 11. Evolución en LISA del número de artículos en español por macroáreas temáticas: porcentaje respecto al total en español 
la impresión que los investigadores españoles, al no poder competir en este campo, han dirigido estratégicamente sus esfuerzos a otros en los que podían conseguir una mayor efectividad.

Cuando se comparan los gráficos de evolución de los macrosectores temáticos a nivel internacional (figura 9) y español (figura 10, valores absolutos; figura 11, porcentajes), se constata la evolución general descrita en los párrafos anteriores; y en particular el efecto de algunos hitos significativos comunes, como el impacto de internet y la web en los años noventa. Desde un punto de vista más local, el pico de producción recogido en LISA parece coincidir con el esfuerzo de investigación que acompaña al tardío pero contundente despegue académico de la información y documentación en España, especialmente durante los años noventa (Jiménez-Contreras; De-Moya-Anegón, 1997; Delgado-López-Cózar, 2002). También constituye un aspecto distintivo la fuerte estructura de ondas en la bibliografía en español en el nuevo milenio. Da la impresión de que la situación socioeconómica afectó fuertemente a la investigación en español, pues se aprecian bajones considerables después de 2008 - "crack del ladrillo"- y 2013 -en torno a la Ley "Wert" 8/2013 y otras medidas de ajuste presupuestarios que se aplicaron al sistema español de ciencia y tecnología-, sin duda a través de variables como la promoción de científicos y académicos, y la disponibilidad de fondos para la investigación.

En lo que se refiere exclusivamente a la investigación en español, también se aprecian muy bien en la figura 11:

- la pérdida de interés relativo por el impacto de la tecnología -que se da ya como un factor estable y se integra en investigaciones especializadas-;

- el mantenimiento de la preocupación por las cuestiones transversales, y por las bibliotecas, muy bien representadas desde 1994; $y$,

- al contrario, se constata el despliegue definitivo de las documentaciones especializadas, y

- el crecimiento sostenido de la informetría, en consonancia clara con las tendencias internacionales.

A continuación se analizan con detalle los conjuntos temáticos de cada macroárea:

\subsection{Los grandes temas transversales: aspectos educacionales, sociales, legales y comportamentales en la investigación LIS española}

Dentro de la tendencia global a la relativa pérdida de interés por los temas transversales en la investigación en español, hay diferencias notables entre los temas de esta área (figura 12). Se aprecia crecimiento en el interés por la preservación documental, la organización, los aspectos teóricos y epistemológicos, la organización del conocimiento y los aspectos éticos y legales. Por el contrario, ha decrecido el interés por los estudios de usuarios y comportamiento informacional, y especialmente por las cuestiones ligadas al desarrollo social. Prácticamente estable, aunque con una ligera tendencia decreciente ha permanecido el interés por los aspectos educativos y profesionales de LIS.

Los aspectos teóricos y epistemológicos aparecen con fuerza entrada la década de los noventa, coincidiendo con el despegue de los departamentos universitarios de Biblioteconomía y Documentación (García-Marco, 2013), y han tenido un interés sostenido tras el cambio de siglo, en línea con la tendencia notada por Delgado-López-Cózar (2002, p. 131).

Los estudios de usuarios, uno de los frentes de investigación en LIS más consolidados a nivel internacional (Åström, 2007), han sido cultivados con fuerza por los autores en español desde finales de los setenta y principios de los ochen-

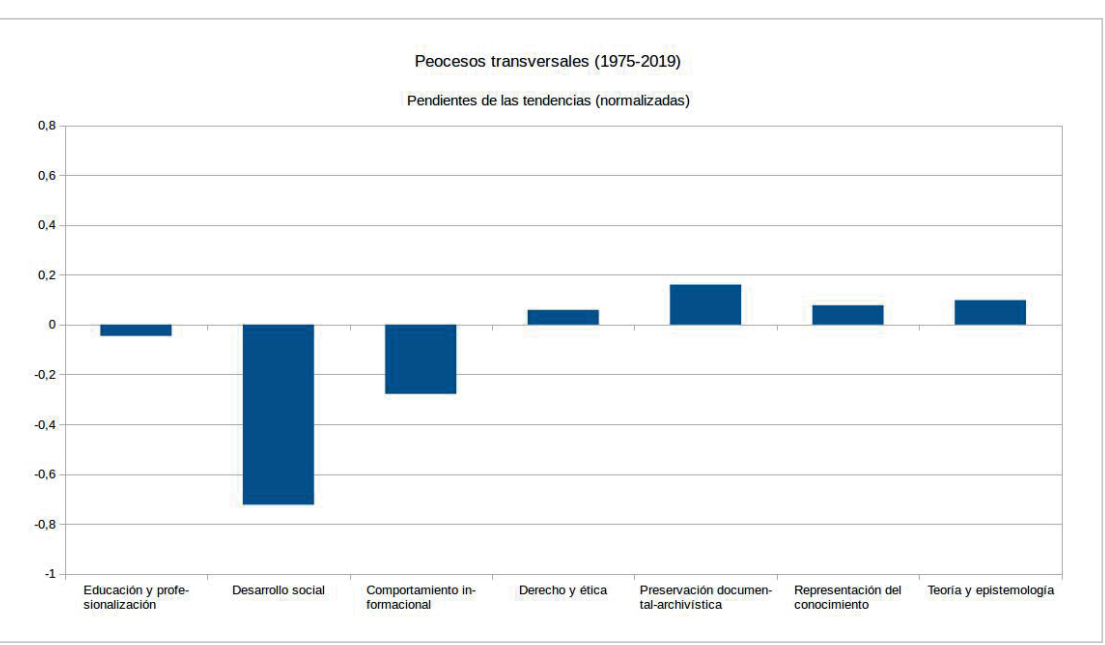

Figura 12. Pendientes de las rectas de regresión de la investigación española sobre los grandes temas transversales (1978-2014)

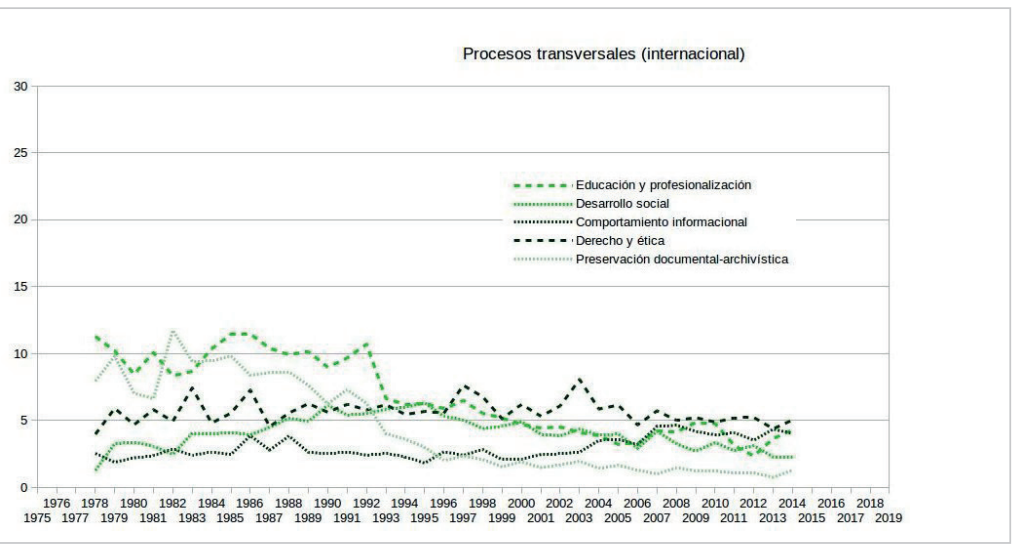

Figura 13. Evolución por años de los grandes temas transversales (internacional) 
ta, con un interés renovado desde finales de los ochenta, para disminuir progresivamente desde el cambio de siglo, posiblemente por la internacionalizacion de las publicaciones en el área y la especialización de los enfoques. Tampoco destaca especialmente el grupo de artículos relacionados con la organización del conocimiento, un grupo que no apareció en el estudio internacional de 2017, y que, sin embargo, ha protagonizado un salto muy importante en los últimos tres años, coincidiendo con el renovado interés por los lenguajes documentales como base para la construcción de ontologías ligeras y mapeos semánticos para el intercambio y descubrimiento de recursos en la web semántica.

Los aspectos legales han sido objeto de un interés continuo, pero que salta al ritmo de los grandes momentos legislativos: la Constitución española en 1978, y las leyes de régimen local, autonomía universitaria, patrimonio y bibliotecas públicas estatales y autonómicas en los años ochenta. Ha sido, sin embargo, notable el despegue de las investigaciones en los aspectos legales y éticos de la información, sobre todo a partir de 2010. Si para la tendencia internacional el pico se produjo en 2003, un año después de la E-government act estadounidense; la bibliografía en español sobre el tema recogida en LISA despegó en 2007, en torno a la promulgación española del Reglamento de desarrollo de la Ley Orgánica 15/1999, de 13 de diciembre, de protección de datos de carácter personal (LOPD), y alcanzó un pico en 2011-2012, en torno al esfuerzo máximo de despliegue de las normas españolas de interoperabilidad en las administraciones públicas y del marco europeo de reutilización de información de la información del sector público (Galindo-Ayuda, 2014).

Por su parte, los temas relacionados con la formación y el desarrollo profesional, prácticamente no experimentaron crecimiento en el panorama internacional, después de un interés fuerte pero con mucha concentración de publicaciones en varios picos entre los años 1978 y 1992. Sin embargo, los artículos en español se han recuperado notablemente en la primera década del milenio coincidiendo con la reconfiguración española de los estudios en información y documentación (Aneca, 2004) en el marco del proceso de Bolonia y la notable crisis en el ingreso de nuevos estudiantes que ha acompañado a la reubicación de los estudios desde el campo de las humanidades en el que nacieron al de las ciencias sociales bajo una nueva marca (García-Marco, 2008; 2013; Ortiz-Repiso; Calzada-Prado; Aportela-Rodríguez, 2013). Esta crisis se produjo antes en los países anglosajones, en los que se aprecia el problema de fondo del ajuste a la revolución web y a los nuevos paradigmas en ciencias humanas y sociales (Cronin, 2005).

Por el contrario, la preocupación por el papel de LIS en el desarrollo social ha experimentado una caída muy importante y constante con el cambio de siglo, después de haber sido extraordinariamente importante durante la Transición y los años noventa (globalización), y ha quedado subsumida en abordajes más técnicos. Sin embargo, el análisis LDA de las publicaciones en español ha aflorado un grupo específico dentro de la investigación bibliotecaria que está especialmente centrado en el papel social de la biblioteca y del bibliotecario. Este grupo experimenta precisamente su crecimiento a partir del cambio de siglo, coincidiendo con la bajada del grupo transversal dedicado al desarrollo social. Así pues, parece que la reflexión sobre el desarrollo social y político se ha refugiado especialmente en el campo de la investigación bibliotecológica.

La reducida importancia del tema preservación que se aprecia durante gran parte del periodo es compatible también con el bajo número de artículos sobre archivística en 2012-2014 que se constata en el estudio de Ferran-Ferrer et al. (2017). Como bien señalan estos autores, esto se debe a que las investigaciones archivísticas se recogen en otras revistas especializadas no siempre indizadas en las bases de datos en cuestión, aunque revistas como el Boletín de la Anabad o Ibersid, que sí se incluyen en LISA, contienen buen número de trabajos de este campo. De hecho, desde el cambio de siglo se han incrementado notablemente los estudios que han quedado clasificados en este grupo, como se puede apreciar en la figura 14, lo que podría indicar una tendencia a la mayor integración disciplinaria de las denominadas en España significativamente "ciencias de la documentación".
La preocupación por el papel de LIS en el desarrollo social en España ha experimentado una caída muy importante y constante con el cambio de siglo 


\subsection{Impacto tecnológico: importando en LIS los avances de las tecnologías de la información y la comuni- cación}

Saracevic (1999) describió acertadamente la LIS como un campo que evoluciona al ritmo de la tecnología disponible -technology-driven-. La aplicación de nuevas tecnologías y técnicas inspira muchas de las preguntas y trabajos de investigación. El despliegue de la Internet y su explosión gracias a la facilidad de uso de la web han impactado de forma decisiva la investigación en el campo, pues se ha convertido en un intermedio en que se hacen disponibles actualmente casi todas las otras fuentes y medios de información. Esto es también cierto para las bases de datos, los catálogos de bibliotecas y las propias colecciones de documentos (Åström, 2007, p. 956).

La tecnología ha tenido una importancia creciente a lo largo de todo el periodo tanto en la bibliografía en español como en el conjunto de LISA (figura 16 y figura 17). Los grupos que ha proporcionado el análisis $L D A$ para la bibliografía en español tienen una configuración diferente de la que reveló para el conjunto de LISA. No hay un grupo distintivo de estadística avanzada, y el de redes de comunicación tiene una composición más general, por lo que se le ha denominado "cambio tecnológico". Los tres grupos muestran una tendencia muy estable en todo el periodo, ligeramente descendente en el caso de la representación y procesamiento automáticos de la información, y ligeramente ascendente en el de búsqueda online (figura 15).

La representación automática del conocimiento ha experimentado un interés continuo, pero con fuertes picos. Sin embargo, desde el surgimiento de la Web y los navegadores (1993-1996), la búsqueda online despegó con mucha intensidad, alcanzando un pico en 2012 y uno intermedio en 2002, que coincide significativamente con la burbuja de las empresas "punto com". Efectivamente, la búsqueda online constituyó en sus comienzos un tema muy técnico que requería conocimientos de programación, redes y hardware muy avanzados. Sin embargo, la estandarización en los procesos de búsqueda, la Web y los avances en las interfaces de usuario lo han terminado por hacer accesible al gran público, al menos en sus aspectos más elementales.

Igual que ocurre a nivel internacional, el interés por el cambio tecnológico y las redes de comunicación en LISA, presente en español desde los años ochenta, despega justamente a partir de 1996, cuando la Web ya está consolidada, y se aprecia una caída a partir de 2012, tras la crisis de 2007 y con un cierto retraso respecto a la tendencia internacional.

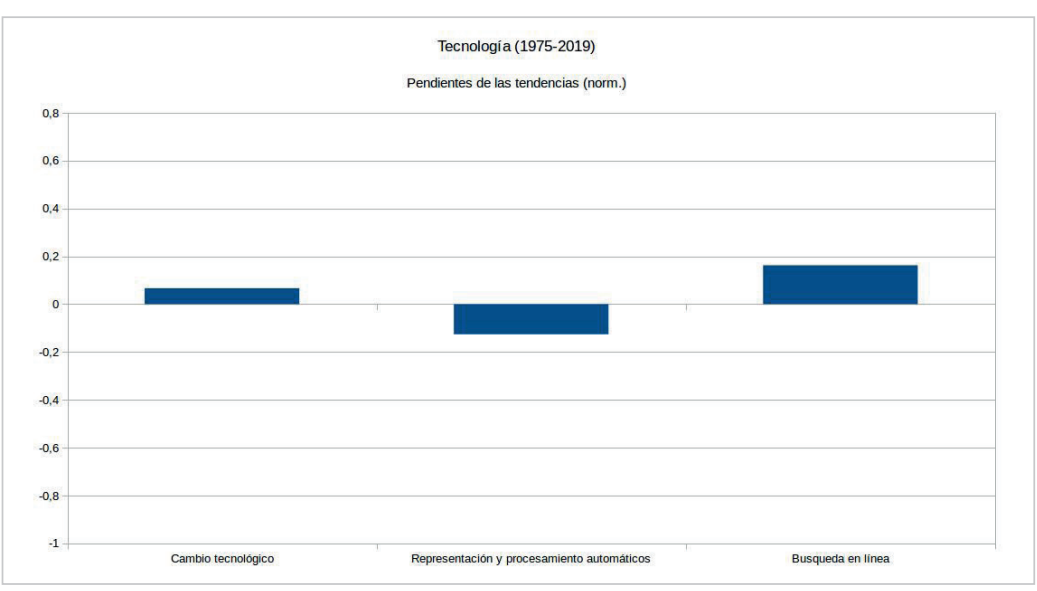

Figura 15. Pendientes de las rectas de regresión de la investigación española sobre tecnologías

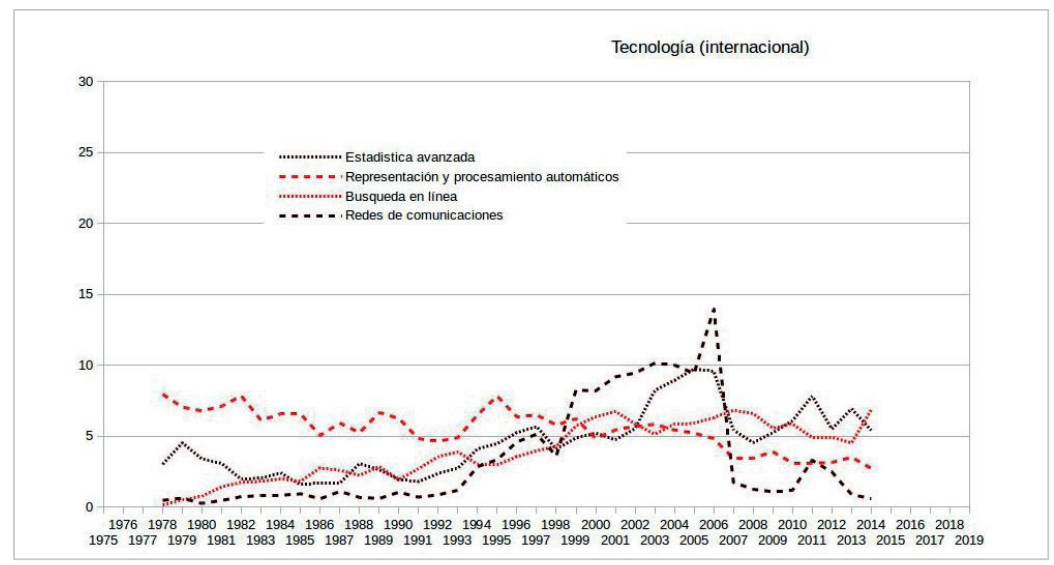

Figura 16. Evolución por años de la macroárea tecnologías (internacional)

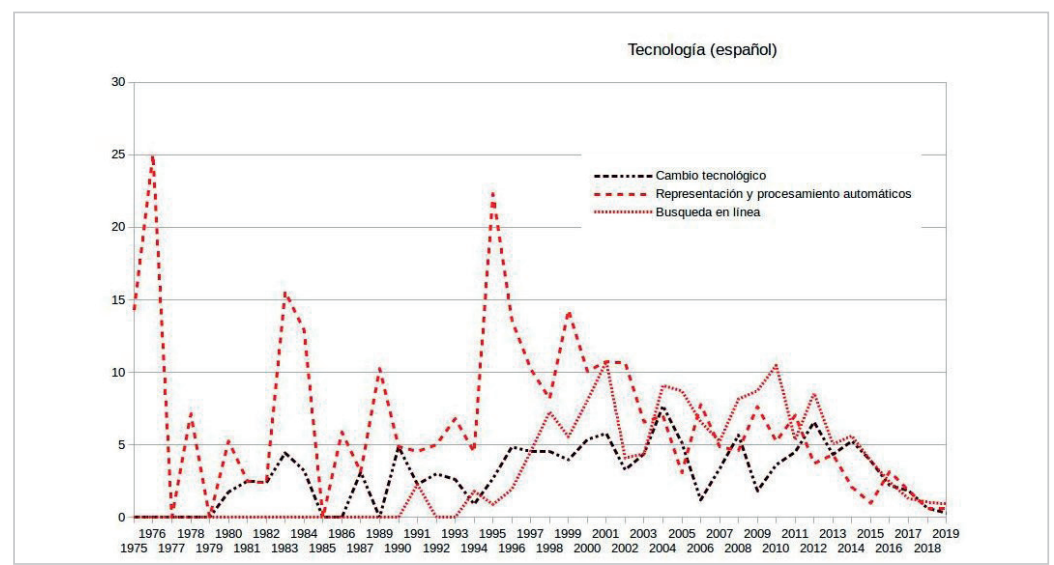

Figura 17. Evolución por años de la macroárea tecnologías (en español) 


\subsection{Las bibliotecas como campo clave de la institucionalización de la LIS}

El macroárea de investigación sobre bibliotecas en español tiene una configuración diferente a la que se detectó para la investigación internacional en 2017 (Figuerola; García-Marco; Pinto, 2017). No aparece un grupo de servicios de referencia, $y$, sin embargo, emerge otro de aspectos políticos y sociales.

El conjunto de aspectos políticos y sociales experimenta una tendencia ligeramente positiva, que se incrementa notablemente después de los años de más impacto de la crisis económica. Por su parte, gestión de bibliotecas y servicios experimenta un fuerte interés en los años 1992-1994, para mantenerse estable hasta 2015.

La catalogación ha perdido interés a nivel internacional, pero en la investigación en español ocurre lo contrario. El interés comienza también después de la revolución de la Web con picos fuertes y un interés consolidado desde 2003 que culmina en 2008. Este ciclo coincide con la incorporación en el ámbito hispánico de la revolución normalizadora que han exigido los recursos electrónicos y ha potenciado las posibilidades que ofrece la web semántica. Es importante recordar en este caso que la investigación sobre desarrollo y aplicación de formatos bibliográficos conservó un carácter bastante local (nacional) hasta que los estadounidenses consiguieron, en el marco de un notable esfuerzo de concertación internacional, la dirección indiscutible del sector, con un hito en la creación de MARC21 en 1999 que coincide aproximadamente con la perdida de interés relativo tanto a nivel internacional como español. El énfasis de todas formas estaba ya girando hacia la compatibilidad tras la invención de la Web, que rompía la compartimentalización de centros, sistemas y redes, y globalizaba los recursos electrónicos en el marco del acceso integrado que promueven los grandes buscadores. El esfuerzo de normalización internacional no ha desparecido: ha sostenido un núcleo de investigación muy productivo, pero muy internacionalizado y normalizado, orientado a datos abiertos en la web semántica (Kroeger, 2013; McCallum, 2017).

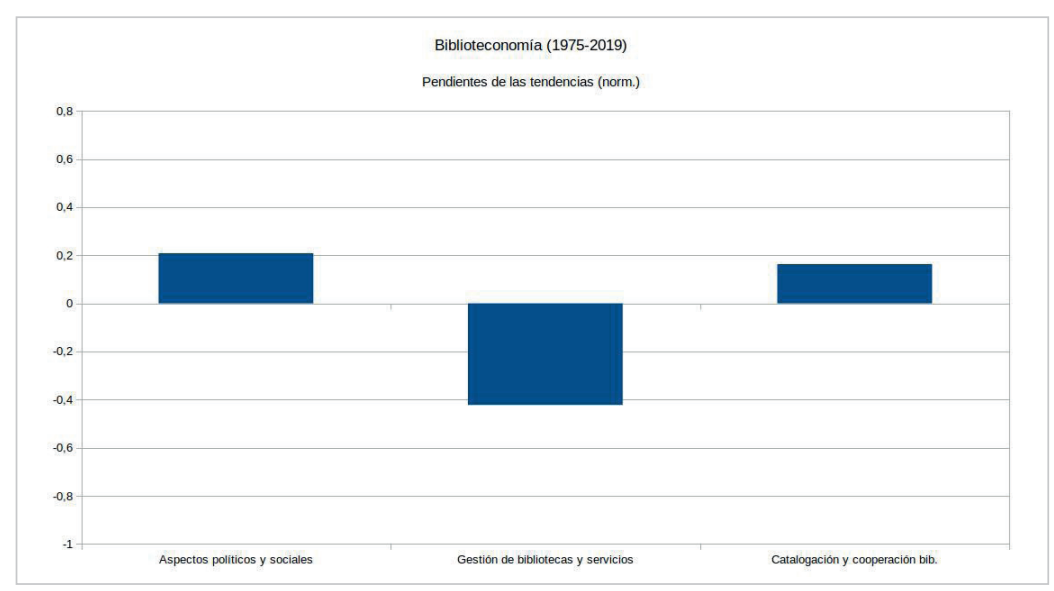

Figura 18. Comparación de las pendientes de las rectas de regresión de la investigación española e internacional sobre bibliotecas

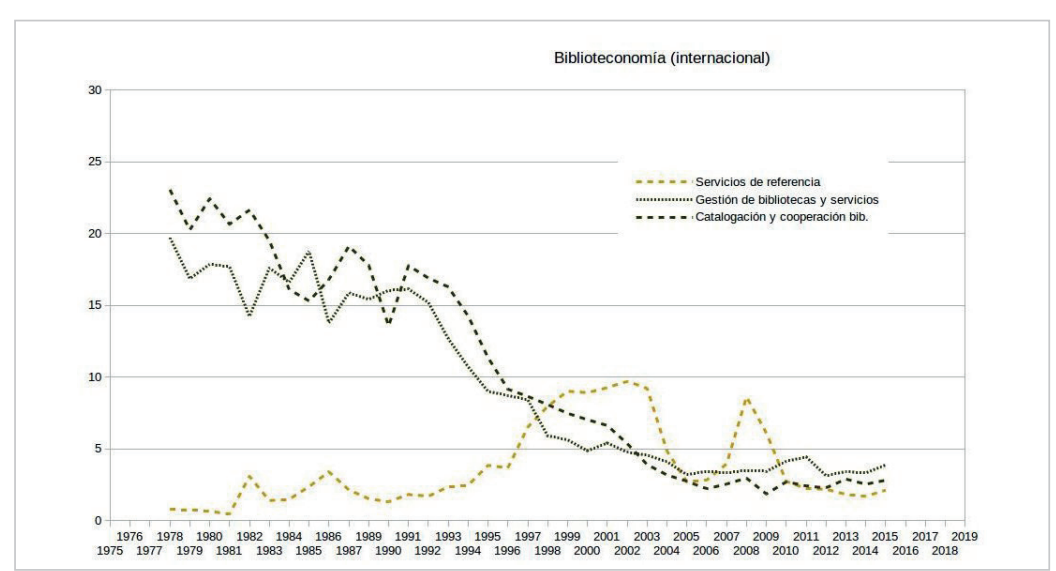

Figura 19. Evolución por años de la macroárea bibliotecas (internacional)

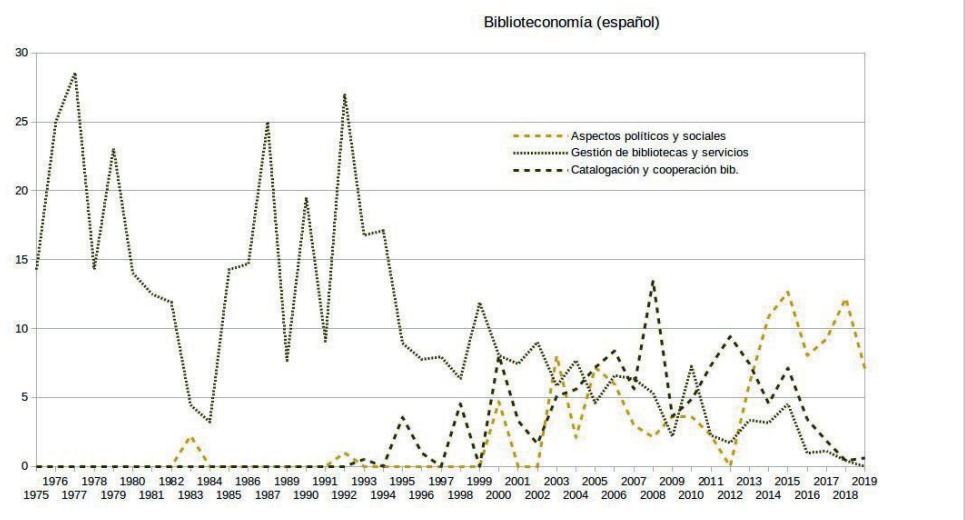

Figura 20. Evolución por años de la macroárea bibliotecas (en español)

\subsection{Despegue de las documentaciones especializadas}

Uno de los principales resultados de una investigación anterior sobre la evolución de los artículos evaluados por pares en LISA sobre LIS (Figuerola; García-Marco; Pinto, 2017) fue constatar que solo el campo de las documentaciones especializadas -que allí incluía la informetría- ha experimentado un crecimiento notable, triplicándose. En dicho trabajo se pueden consultar de forma detallada las conclusiones obtenidas para cada uno de los conjuntos temáticos. 
La aplicación de LDA a los registros de LISA en español ha producido la emergencia de grupos temáticos nuevos, en particular, uno de redes sociales y otro de gestión de la información y el conocimiento en instituciones de educación superior, con el objeto de facilitar su evaluación y mejora. Por otra parte, desaparece el específico de información empresarial o business information. El interés por las redes sociales surge pronunciadamente en 2015 hasta prácticamente ponerse en cabeza, de forma paralela también al intenso y reciente despegue en las investigaciones orientadas a mejorar la educación superior.

La especialización temática en los campos de la información empresarial, legal, educativa, mediática y patrimonial constituye un signo de madurez disciplinar, en la línea de lo que ha ocurrido, por ejemplo, en los campos de las ciencias de la salud o las ingenierías. Los grandes avances tecnológicos en el campo de la computación y la telemática aplicados a la información y la documentación, y la continuación en los trabajos de normalización de procesos, normativa y formatos han constituido el sustrato de este enorme avance. Aunque el efecto no es tan espectacular como en la producción internacional, las investigaciones aplicadas en español referidas en LISA aumentan notablemente desde 1993, coincidiendo con los avances que se producen en la difusión de internet. En el caso de la bibliografía en español son fácilmente observables los brotes de interés en información mediática que se producen tras la invención de los navegadores (19951996) y la explosión de las redes sociales (2005), las dos tecnologías que han revolucionado el mundo de los medios en las últimas décadas (Hobbs; Jensen, 2009; Kellner; Share 2005), a pesar de que la producción total no es alta (Sánchez-Vigil; Marcos-Recio; Olivera-Zaldua, 2015). Si se considera el campo de la comunicación en su conjunto, el estudio muestra datos compatibles para finales del periodo estudiado en este artículo (2012-2014), con un 9\% de los artículos prospectados (Ferran-Ferrer; Guallar; Abadal; Server, 2017).

En el despliegue de las documentaciones especializadas, las pendientes de las rectas de regresión para la producción científica internacional y en español recogida en LISA son muy semejantes para los diferentes conjuntos temáticos, concretamente para la información patrimonial y humanística, mediática, educacional, empresarial y la interespecialidad gestión del conocimiento. Sin embargo, hay una excepción: la información sanitaria, cuya pendiente experimenta una caída significativa, después de haber sido claramente la documentación aplicada pionera desde 1975, con gran diferencia sobre todas las demás. Esto puede deberse a que posiblemente -lo mismo que ocurre con la informetría- la investigación sanitaria esté afectada por la creciente publicación de investigadores españoles en revistas internacionales no españolas, pues en el campo de la información en ciencias de la salud la investigación española está muy internacionalizada, según revelan Jiménez-Contreras, Delgado-López-Cózar y Ruiz-Pérez (2006, p. 382) y De-la-Moneda (2003).

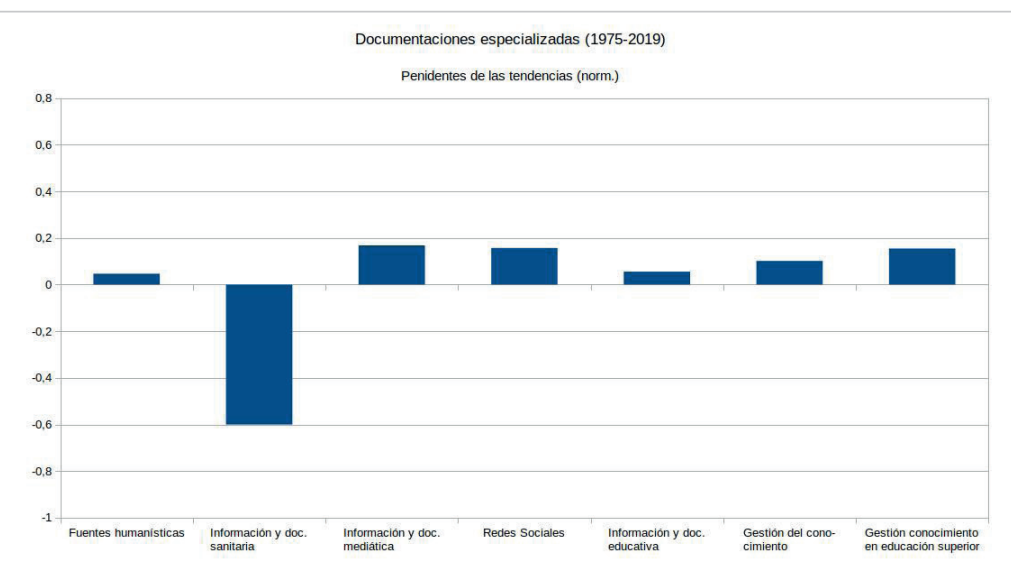

Figura 21. Comparación de las pendientes de las rectas de regresión de la investigación española e internacional sobre documentaciones especializadas

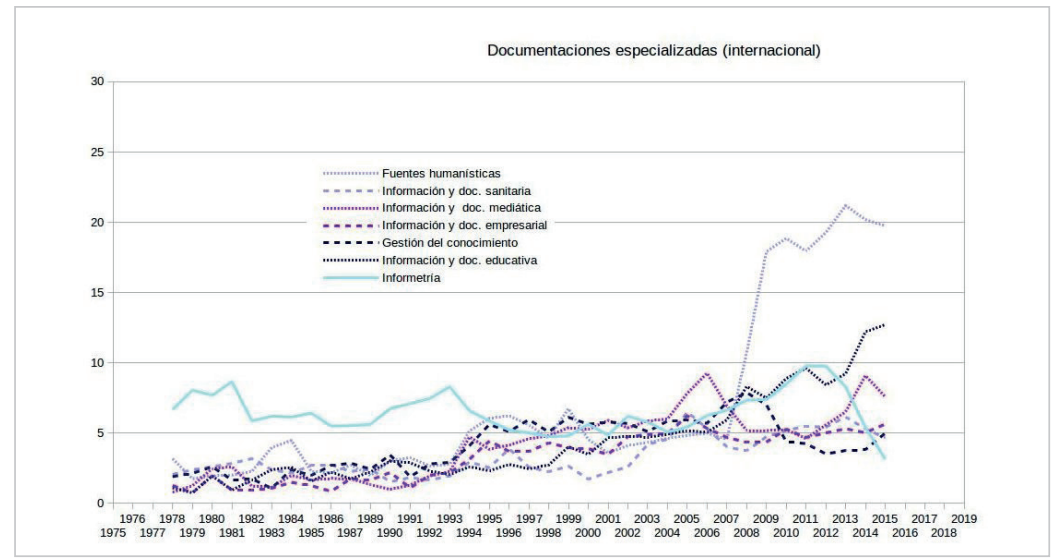

Figura 22. Evolución por años de las documentaciones especializadas (internacional)

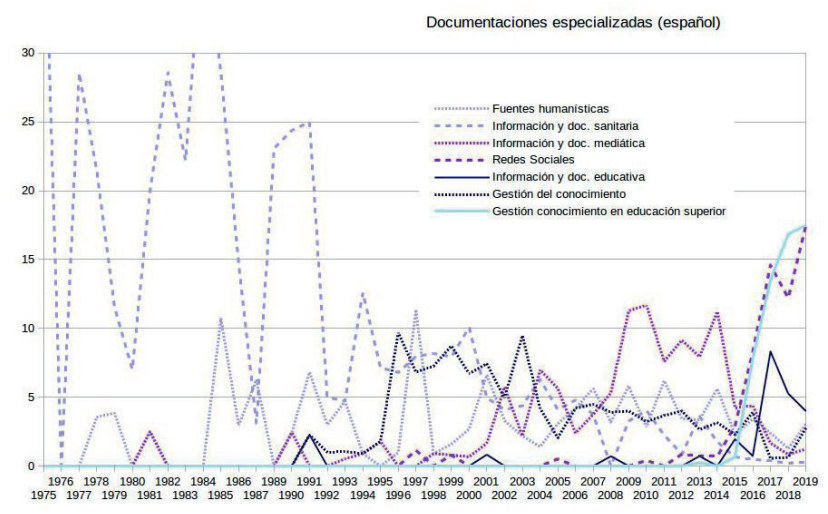

Figura 23. Evolución por años de las documentaciones especializadas (en español) 


\subsection{Investigación informétrica}

En español, la producción sobre informetría y bibliometría se constituye como un macroárea de investigación propia, que agrupa cuatro conjuntos empíricos. La importancia de la investigación bibliométrica en nuestro país ha sido notada por todos los autores que se han ocupado de la producción científica sobre LIS en España, y ha sido contextualizada con precisión por Jiménez-Contreras y De-Moya-Anegón (1997) y Delgado-López-Cózar (2000; 2002): la investigación española se puede dividir en tres frentes de investigación -el bibliotecario, de carácter muy práctico, el bibliométrico, compacto e internacionalizado y el universitario, muy heterogéneo-. El frente informétrico se apoya en la temprana trayectoria del Cindoc del Consejo Superior de Investigaciones Científicas en Madrid y del Instituto de Estudios Históricos y Documentales sobre la Ciencia de Valencia, hoy Instituto de Historia de la Medicina y de la Ciencia López Piñero. El principal órgano de comunicación de esta comunidad de investigadores ha sido y sigue siendo la Revista española de documentación científica (Pérez-Álvarez-Ossorio, 1997; Jiménez-Hidalgo, 2007; Ríos-Hilario, 2011; REDC, 2014).

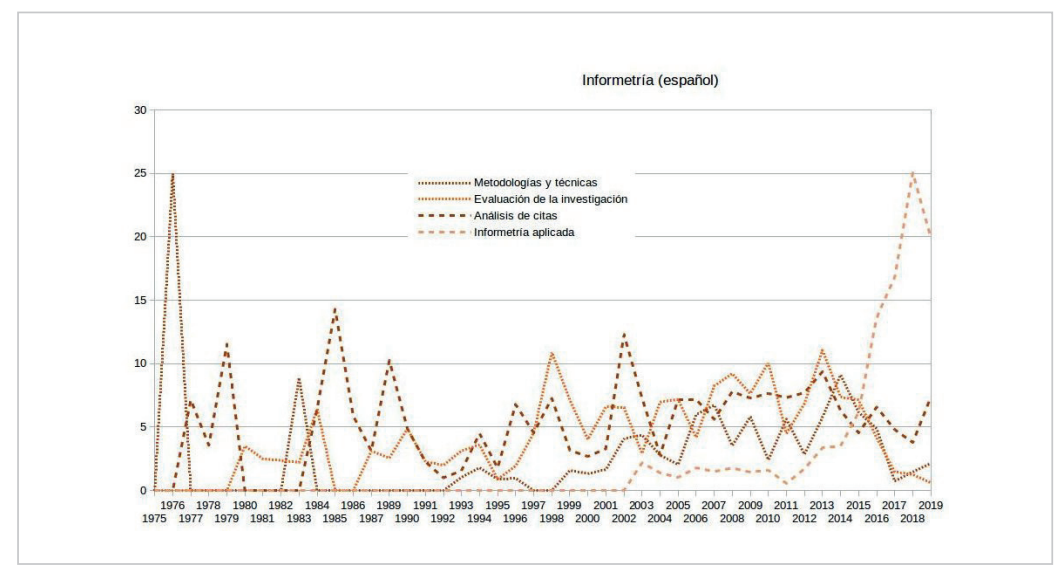

Figura 24. Evolución por años del área de informetría (en español)

Esa semilla ha fructificado en varios gru-

pos de investigación en Granada, Madrid y Salamanca, entre otros lugares, y ha retroalimentado los primeros núcleos, manteniendo en su conjunto una trayectoria de alto nivel internacional (Pandiella-Dominique; García-Zorita; Sanz-Casado, 2019).

Sin embargo, el enorme protagonismo de la bibliometría en la mitad del periodo ha dado paso a una producción en español algo menor tanto en términos absolutos como relativos, mientras que a nivel internacional ha seguido creciendo (REDC, 2014). Esta aparente divergencia se debe fundamentalmente a que gran parte de la producción de este campo se publica hoy en día en revistas internacionales en inglés, como Scientometrics, Jasist o Journal of informetrics, entre otras; no a que haya disminuido la producción de científicos españoles e iberoamericanos sobre el tema, antes al contrario (Ardanuy, 2012). Con todo, el análisis para final del periodo estudiado realizado por Ferran-Ferrer et al.(2017) confirmaba que se trataba del segundo tema en importancia, con el $14 \%$ del total de los artículos analizados, y el primero en artículos de investigación, con el 19\%.

Se puede observar que, dentro de la tendencia positiva -aunque ligera- apreciable en todos los grupos temáticos, destaca el crecimiento más intenso de la informetría aplicada, constituida por investigaciones que aplican métodos estadísticos a la resolución de problemas no

\section{La catalogación ha perdido interés a ni- vel internacional, pero en la investiga- ción en español ocurre lo contrario} tradicionalmente bibliométricos (figura 25). Desde 2014 este grupo despega verticalmente, correlacionando con la formación semejante que se aprecia en las investigaciones sobre redes sociales, que ya se ha analizado anteriormente. En el caso contrario están las publicaciones sobre metodologías y técnicas, que tuvieron un interés muy alto en el año 1976 y descienden al final del periodo estudiado, aunque desde 2001-2002 han recibido un interés sostenido hasta 2014.

\section{Conclusiones}

Los estudios de evolución de la investigación en LIS realizados sobre la base de datos LISA tienen el interés de su amplia cobertura temporal, también para las revistas en español en una época en la que quedaban fuera de los índices de citas existentes, fundamentalmente los producidos por el Institute for Scientific Information, surgido en 1960. Por el contrario, tienen un sesgo editorial que resulta menos pronunciado en los índices de citas, por lo que se pueden considerar fuentes que pueden ofrecer análisis complementarios. 
En general, la aplicación del LDA a la bibliografía referida en español ha producido resultados coherentes con el estudio de tendencias globales internacionales (Figuerola; García-Marco; Pinto, 2017), con los estudios de Prebor (2007; 2010) sobre las tesis de ProQuest Digital Dissertations clasificadas en los campos 'Library Science' e 'Information Science', y con las numerosas investigaciones de los autores españoles que se han ocupado de la cuestión y que se han referido en estas páginas. En este sentido, Olmeda-Gómez (2013, p. 10) señala que la adhesión de nuevos trabajos y nuevos investigadores a la red de citación mundial en LIS no ha

"afectado a los tamaños de las producciones elaboradas por la élite del dominio. Sencillamente, se han adherido."

Esta afirmación parece que se cumple también en lo referente a la evolución de los temas.

La orientación general de los resultados para la bibliografía en español de LISA se alinea con la afirmación de Tuomaala, Järvelin y Vakkari (2014) de que los intereses temáticos de investigación han girado en los últimos cincuenta años desde el enfoque en las bibliotecas y las organizaciones informacionales a los usuarios y el desarrollo de sistemas específicos, que en este artículo hemos denominado áreas de aplicación y que incorporaría la biblioteconomía, las documentaciones especializadas, y la informetría y bibliometría. Sin embargo, posee por otra parte numerosas características propias dignas de destacar tanto en cuanto a los conjuntos temáticos que han surgido como a las diferencias en tamaños y tendencias.

En cuanto a los conjuntos empíricos, el análisis LDA ha aflorado algunos grupos específicos, no necesariamente grandes en tamaño, pero que comparten un vocabulario común. Se trata en concreto de las investigaciones en organización del conocimiento, los aspectos teóricos y epistemológicos, el papel social de la biblioteca y los bibliotecarios, las redes sociales, y la gestión de la información y el conocimiento aplicados a la mejora de la educación superior. Es muy notable la aparición de cuatro conjuntos temáticos para bibliometría e informetría -metodologías y técnicas, estudios de citas e impacto, evaluación de la investigación, e informetría aplicada (interdisciplinar)-, lo que muestra la importancia de la investigación sobre estas cuestiones en español. Por el contrario, desaparecen los grupos de estadística avanzada, servicios de referencia, e información y documentación empresarial. También toman un cariz diferente algunos grupos. Así, el de catalogación y cooperación bibliotecaria tiene un perfil muy orientado a las bibliotecas y repositorios digitales; y el de redes de comunicación ha quedado embebido en uno más general de cambio tecnológico, que muestra un vocabulario más general que en en el enfoque internacional.

En cuanto al tamaño y tendencias diferenciales de la investigación en español, destaca -además de la importancia de la investigación bibliométrica e informétrica, que se ha discutido ya extensamente en el apartado anterior- una preocupación sostenida en el tiempo por las cuestiones de carácter general.

Este interés por cuestiones más generales se suma a otros resultados encontrados -en particular, el interés algo más reducido por el impacto de las tecnologías, la constatación de que las documentaciones especializadas han crecido en general menos que en el panorama internacional, el crecimiento estable de la investigación sobre bibliotecas, una demora de cinco años en el pico de preocupación por las cuestiones legales y éticas, y un sostenido interés por las cuestiones educativas y de desarrollo profesional, que se comentará después- para indicar en su conjunto un cierto retraso en la evolución de la investigación española respecto a los países anglosajones.

Dicho retraso fue apuntado en el quicio del milenio por los principales investigadores españoles que se han ocupado del tema (Jiménez-Contreras; De-Moya-Anegón, 1997; Cano, 1999; Delgado-López-Cózar, 2000; 2002), aunque a finales del periodo varios autores constatan también indicadores claros de la madurez de la investigación en español (Ríos-Hilario; Travieso-Rodríguez, 2011; Ardanuy, 2012; Guallar et al., 2017) a pesar de una falta de estructuración consistente de sus temas en la larga duración, con la excepción ya citada de la investigación bibliométrica (Olmeda-Gómez; Ovalle-Perandones; Perianes-Rodríguez, 2017) y biomédica.

Por su parte, el interés por la formación y el desarrollo profesional -estancado en el ámbito internacional desde principios de los años noventa- se aprecia de nuevo en la década primera del milenio coincidiendo con la reconfiguración española de los estudios en información y documentación dentro del llamado proceso de Bolonia, seguido poco después por el debate sobre la crisis de los estudios universitarios, que acontece antes en el mundo anglosajón (Cronin, 2005). Es importante señalar también que el interés por los aspectos de desarrollo social y político se ha refugiado en el campo de la investigación bibliotecológica a partir del cambio de siglo, probablemente muy en relación con el impacto social y presupuestario de la crisis económica.

En cuanto a perspectivas de trabajo futuro, además de las más obvias (replicar el estudio en periodos posteriores, en bases de datos diferentes y en subdisciplinas más específicas de LIS), ha surgido como un tema interesante de investigación futu- 
ra la comprobación de hasta qué punto la filiación de los investigadores ofrece también una posibilidad indirecta para estudiar la evolución temática de LIS, puesto que la especialidad temática de los centros de adscripción de los investigadores suele resultar relativamente fácil de determinar a grandes rasgos (Jiménez-Contreras; Delgado-López-Cózar; Ruiz-Pérez, 2006).

Otra cuestión de alcance más general de gran interés para el trabajo futuro es la elaboración de metodologías para hacer más objetivo el proceso de categorización y taxonomización de los conjuntos temáticos hallados mediante la aplicación de LDA. Esta es una cuestión difícil más allá de las medidas más obvias: fundamentalmente el añadido de cautelas sobre el número de jueces, el proceso de consenso y su documentación.

Por último, se ha podido comprobar que el interés de la aplicación de estas técnicas de datos masivos (big data) e inteligencia artificial al estudio del desarrollo y configuración de la ciencia y de las disciplinas científicas reside, entre otras cosas, en que obliga a los investigadores a contrastar sus hipótesis y su marco epistémico sobre las disciplinas en las que trabajan con los resultados objetivos que resultan del mapeo $L D A$, forzando un proceso de pensamiento lateral y de autocrítica muy productivo en un campo, como es la epistemología científica, muy dominado por los paradigmas en los que se han educado los investigadores y a los que se adscriben (Kuhn, 1970).

\section{Referencias}

Aneca (2004). Título de grado en información y documentación: libro blanco. Madrid: Agencia Nacional de Evaluación de la Calidad y la Acreditación.

Ardanuy, Jordi (2012). "Scientific collaboration in library and information science viewed through the Web of Knowledge: The Spanish case". Scientometrics, v. 90, pp. 877-890.

https://doi.org/10.1007/s11192-011-0552-1

Åström, Fredrik (2007). "Changes in the LIS research front: Time-sliced cocitation analyses of LIS journal articles, 19902004". Journal of the American Society for Information Science and Technology, v. 58, n. 7, pp. 947-957.

https://doi.org/10.1002/asi.20567

Blessinger, Kelly; Frasier, Michele (2007). "Analysis of a decade in library literature: 1994-2004". College \& research libraries, v. 68, n. 2.

https://doi.org/10.5860/crl.68.2.155

Blessinger, Kelly; Hrycaj, Paul (2010). "Highly cited articles in library and information science: An analysis of content and authorship trends". Library \& information science research, v. 32, pp. 156-162.

https://doi.org/10.1016/j.lisr.2009.12.007

Cano, Virginia (1999). "Bibliometric overview of library and information science research in Spain". Journal of the American Society for Information Science, v. 50, n. 8, pp. 675-680.

https://doi.org/10.1002/(SICI)1097-4571(1999)50:8<675::AID-ASI5>3.0.CO;2-B

Chowdhury, Gobinda; Koya, Kushwanth (2017). "Information practices for sustainability: Role of iSchools in achieving the UN sustainable development goals (SDGs)". Journal of the Association for Information Science and Technology, v. 68, pp. 2128-2138. https://doi.org/10.1002/asi.23825

Corona-Lisboa, José-Luis (2018). “Investigación cualitativa: fundamentos epistemológicos, teóricos y metodológicos”. Vivat academia, n. 144, pp. 69-76.

http://doi.org/10.15178/va.2018.144.69-76

Cronin, Blaise (2005). "An I-dentity crisis? The information schools movement". International journal of information management, v. 25, pp. 363-365.

https://doi.org/10.1016/j.ijinfomgt.2005.04.007

De-la-Moneda-Corrochano, Mercedes (2003). Análisis bibliométrico de la producción bibliográfica española en biblioteconomía y documentación, 1984-1999. Granada: Universidad de Granada, Departamento de Biblioteconomía y Documentación. https://digibug.ugr.es/handle/10481/40114

Delgado-López-Cózar, Emilio (2000). “Diagnóstico de la investigación en biblioteconomía y documentación en España (19761996)". Revista de investigación iberoamericana en ciencias de la información y la documentación, v. 1, n. 1, pp. 79-93.

Delgado-López-Cózar, Emilio (2002). La investigación en Biblioteconomía y documentación. Gijón: Trea. ISBN: 8497040414.

Ferran-Ferrer, Núria; Guallar, Javier; Abadal, Ernest; Server, Adán (2017). “Research methods and techniques in Spanish library and information science journals (2012-2014)". Information research, v. 22, n. 1, paper 741.

http://InformationR.net/ir/22-1/paper741.html 
Figuerola, Carlos G.; García-Marco, Francisco-Javier; Pinto, María (2017). “Mapping the evolution of library and information science (1978-2014) using topic modeling on LISA". Scientometrics, v. 112, n. 3, pp. 1507-1535. https://doi.org/10.1007/s11192-017-2432-9

Galindo-Ayuda, Fernando (2014). "La regulación de los datos abiertos". Ibersid: revista de sistemas de información y documentación, v. 8, pp. 13-18. https://www.ibersid.eu/ojs/index.php/ibersid/article/view/4169

García-Marco, Francisco-Javier (2008). "La biblioteconomía y la documentación española en la encrucijada". Ibersid: revista de sistemas de información y documentación, v. 2, pp. 307-318.

https://www.ibersid.eu/ojs/index.php/ibersid/article/view/2254

García-Marco, Francisco-Javier (2013). “Educación y aprendizaje de la información y la documentación: raíces, desafíos y líneas de acción". El profesional de la información, v. 22, n. 6, pp. 489-504. https://doi.org/10.3145/epi.2013.nov.01

Guallar, Javier; Ferran-Ferrer, Núria; Abadal, Ernest; Server, Adán (2017). "Revistas científicas españolas de información y documentación: análisis temático y metodológico”. El profesional de la información, v. 26, n. 5, pp. 947-960. https://doi.org/10.3145/epi.2017.sep.16

Hjørland, Birger (2018a). "Library and information science (LIS). Part 1”. Knowledge organization, v. 45, n. 3, pp. $232-254$. https://doi.org/10.5771/0943-7444-2018-3-232

Hjørland, Birger (2018b). "Library and information science (LIS). Part 2". Knowledge organization, v. 45, n. 4, pp. 319-338. https://doi.org/10.5771/0943-7444-2018-4-319

Hobbs, Renee; Jensen, Amy (2009). "The past, present, and future of media literacy education". Journal of media literacy education, v. 1, n. 1, pp. 1-11.

https://digitalcommons.uri.edu/jmle/vol1/iss1/1

Janssens, Frizo; Leta, Jacqueline; Glänzel, Wolfgang; De-Moor, Bart (2006). "Towards mapping library and information science". Information processing and management, v. 42, n. 6, pp. 1614-1642.

https://doi.org/10.1016/j.ipm.2006.03.025

Järvelin, Kalervo; Vakkari, Pertti (1990). "Content analysis of research articles in library and information science”. Library \& information science research, v. 12, pp. 395-421.

Järvelin, Kalervo; Vakkari, Pertti (1993). "The evolution of library and information science 1965-1985: A content analysis of journal articles". Information processing \& management, v. 29, n. 1, pp. 129-144.

https://doi.org/10.1016/0306-4573(93)90028-C

Jiménez-Contreras, Evaristo; Delgado-López-Cózar, Emilio; Ruiz-Pérez, Rafael (2006). “Producción española en biblioteconomía y documentación con visibilidad internacional a través del Web of science (1995-2004)". El profesional de la información, v. 15, n. 5, pp. 373-383.

http://www.elprofesionaldelainformacion.com/contenidos/2006/septiembre/6.pdf

Jiménez-Contreras, Evaristo; De-Moya-Anegón, Félix (1997). “Análisis de la autoría en revistas españolas de biblioteconomía y documentación, 1975-1995". Revista española de documentación científica, v. 20, n. 3, pp. 252-266.

https://doi.org/10.3989/redc.1997.v20.i3.581

Jiménez-Hidalgo, Sonia (2007). "Análisis de la autoría en la Revista española de documentación científica (1997-2005)". Revista española de documentación científica, v. 30, n. 3, pp. 305-322.

https://doi.org/10.3989/redc.2007.v30.i3.387

Kawalec, Anna (2013). "Research trends in library and information science based on Spanish scientific publication 2000 to 2010". Malaysian journal of library \& information science, v. 18, n. 2, pp. 1-13. https://pdfs.semanticscholar.org/b4d7/3a76013a4b7c3cf27d58f57e416d9ba24d64.pdf

Kellner, Douglas; Share, Jeff (2005). "Toward critical media literacy: Core concepts, debates, organizations, and policy". Discourse: Studies in the cultural politics of education, v. 26, n. 3, pp. 369-386. https://doi.org/10.1080/01596300500200169

Kroeger, Angela (2013). "The road to Bibframe: The evolution of the idea of bibliographic transition into a post-MARC future. Cataloging \& classification quarterly, v. 51, n. 8, pp. 873-890.

https://doi.org/10.1080/01639374.2013.823584

Kuhn, Thomas S. (1970). The structure of scientific revolutions, $2^{\text {nd }}$ ed. Chicago: University of Chicago Press. ISBN: 9780 226458045 
López-Yepes, José (2015). La ciencia de la información documental: el documento la disciplina y el profesional en la era digital. México, D. F.: Minos Tercer Milenio Editores; Universidad Panamericana. ISBN: 9786074321838

McCallum, Sally (2017). "Bibframe development". JLIS.it, v. 8, n. 3, pp. 71-85.

https://doi.org/10.4403/jlis.it-12415

Mochón-Bezares, Gonzalo; Sorli-Rojo, Ángela (2002). Tesauro de biblioteconomía y documentación. Madrid: Consejo Superior de Investigaciones Científicas. ISBN: 8400080912

https://digital.csic.es/handle/10261/30255

Olmeda-Gómez, Carlos (2013). "La investigación española en documentación en un contexto global”. VI Jornadas de la Red de bibliotecas: investigación española en documentación, ¿en qué liga jugamos?. Madrid, 12 de diciembre de 2013. https://www.cervantes.es/imagenes/File/biblioteca/jornadas/jornada_6/ponencia_carlos_olmeda.pdf

Olmeda-Gómez, Carlos; Ovalle-Perandones, María-Antonia; Perianes-Rodríguez, Antonio (2017). “Co-word analysis and thematic landscapes in Spanish information science literature, 1985-2014". Scientometrics, v. 113, pp. $195-217$. https://doi.org/10.1007/s11192-017-2486-8

Ortiz-Repiso, Virginia; Calzada-Prado, Javier; Aportela-Rodríguez, Ivett M. (2013). “¿Qué está pasando con los estudios universitarios de biblioteconomía y documentación en España?”. El profesional de la información, v. 22, n. 6, pp. 505-514. https://doi.org/10.3145/epi.2013.nov.02

Pandiella-Dominique, Andrés; García-Zorita, Carlos; Sanz-Casado, Elías (2019). "Análisis de la internacionalización de la Revista española de documentación científica: 2010-2015". Revista española de documentación científica, v. 42 , n. 1. https://doi.org/10.3989/redc.2019.1.1594

Pérez-Álvarez-Ossorio, José-Ramón (1997). “Cobertura temática y procedencia institucional de los artículos publicados en la Revista española de documentación científica en sus veinte años de existencia". Revista española de documentación científica, v. 20, n. 3, pp. 290-298.

https://doi.org/10.3989/redc.1997.v20.i3.584

Prebor, Gila (2007). “Information science - Facing social and ethical challenges: Analysis of masters' theses and doctoral dissertations over the past five years (2002-2006) in information science departments worldwide". Journal of information, communication and ethics in society, v. 5, n. 2-3, pp. 253-269.

https://doi.org/10.1108/14779960710837687

Prebor, Gila (2010). "Analysis of the interdisciplinary nature of library and information science". Journal of librarianship and information science, v. 42, n. 4, p. 256-267.

https://doi.org/10.1177/0961000610380820

REDC (2012). "Historia de la Revista española de documentación científica". Revista española de documentación científica, v. 35, n. 4, pp. 521-529.

http://redc.revistas.csic.es/index.php/redc/article/view/761

Ríos-Hilario, Ana-Belén (2001). "Diez años de investigación en la Revista española de documentación científica”. Revista española de documentación científica, v. 24, n. 4, pp. 433-449.

https://doi.org/10.3989/redc.2001.v24.i4.70

Ríos-Hilario, Ana Belén; Travieso-Rodríguez, Críspulo (2013). "Estudio de la Revista española de documentación científica: análisis comparativo de los periodos 1989-1999 y 2000-2010". Revista española de documentación científica, v. 36, n. 3, e016.

https://doi.org/10.3989/redc.2013.3.990

Sánchez-Vigil, Juan-Miguel; Marcos-Recio, Juan-Carlos; Olivera-Zaldua, María (2015). “Producción científica sobre comunicación y medios en las revistas de documentación (2000-2014)". Revista española de documentación científica, v. 38 , n. 4. https://doi.org/10.3989/redc.2015.4.1257

Saracevic, Tefko (1999). "Information science". Journal of the American Society for Information Science, v. 50, pp. 1051-1063. https://doi.org/10.1002/(SICI)1097-4571(1999)50:12<1051::AID-ASI2>3.0.CO;2-Z

Singh, Jagtar; Kerr, Paulette; Hamburger, Esther; Alliance of Civilizations; Communication; Unesco Assistant Director-General for Information (2016). Media and information literacy: reinforcing human rights, countering radicalization and extremism. Paris: Unesco. The Milid yearbook. ISBN: 9789231001871

https://unesdoc.unesco.org/ark:/48223/pf0000246371

Tuomaala, Otto; Järvelin, Kalervo; Vakkari, Pertti (2014). “Evolution of library and information science, 1965-2005: Content analysis of journal articles". Journal of the Association for Information Science and Technology, v. 65, n. 7, pp. 1446-1462.

https://doi.org/10.1002/asi.23034 